Monatsh. Math. 152, 217-249 (2007)

DOI 10.1007/s00605-007-0478-0

Printed in The Netherlands

\title{
Completely empty pyramids on integer lattices and two-dimensional faces of multidimensional continued fractions
}

\author{
By \\ O. N. Karpenkov \\ Leiden University, Leiden, The Netherlands \\ Communicated by J. Schoißengeier
}

Received August 29, 2006; accepted in final form January 30, 2007

Published online June 13, 2007 (C) Springer-Verlag 2007

\begin{abstract}
In this paper we develop an integer-affine classification of three-dimensional multistory, completely empty convex marked pyramids. We apply it to obtain the complete lists of compact two-dimensional faces of multidimensional continued fractions lying in planes at integer distances $2,3,4, \ldots$ to the origin. The faces are considered up to the action of the group of integer-linear transformations.
\end{abstract}

2000 Mathematics Subject Classification: 11H06, 52C07

Key words: Convex polygons, integer lattices, multidimensional continued fractions

\section{Introduction and background}

The main purpose of the present paper is to develop an integer-affine classification of three-dimensional multistory, completely empty convex marked pyramids. We apply it to deduce an integer-linear classification of compact two-dimensional faces of multidimensional continued fractions in the sense of Klein lying in planes at integer distances to the origin greater than one. The classification of twodimensional faces leads to new algorithms of two-dimensional continued fraction calculations. It is also the first step in studying the combinatorial structure of multidimensional continued fractions.

0.1. General definitions. Consider a vector space $\mathbb{R}^{n+1}$ for some $n \geqslant 1$. A point or a vector of $\mathbb{R}^{n+1}$ is called integer if all its coordinates are integers.

Consider some $k$-dimensional plane of $\mathbb{R}^{n+1}$. The intersection of a finite number of closed $k$-dimensional half-planes of the plane is said to be a convex (solid) $k$-dimensional polyhedron if it is homeomorphic to a $k$-dimensional closed disk. For $k=0,1$, or 2 we have a point, a segment, or a convex polygon. Here we consider polyhedra as convex hulls with all their interior points.

Partially supported by NWO-RFBR 047.011.2004.026 (RFBR 05-02-89000-NWO_a) grant, by RFBR SS-1972.2003.1 grant, by RFBR 05-01-02805-CNRSL_a grant, by NWO-DIAMANT grant 613.009.001, and by RFBR grant 05-01-01012a. 
A polyhedron is said to be a convex marked pyramid with some marked face and a vertex outside the plane containing the face if it coincides with the convex hull of the union of the marked vertex and the marked face. The marked face is called the base of the marked convex pyramid and the marked vertex - the vertex of the marked convex pyramid. A polyhedron is called a convex pyramid if some structure of convex marked pyramid can be introduced for it.

A convex polyhedron (polygon, segment) is said to be integer if all its vertices are integer points. A convex (marked) pyramid is said to be integer if it is an integer convex polyhedron.

Definition 0.1. An integer convex polyhedron is called empty if it does not contain integer points different from the vertices of the polyhedron. An integer convex marked pyramid is called completely empty if it does not contain integer points different from the marked vertex and from the integer points of the base.

Two sets in $\mathbb{R}^{n+1}$ are said to be integer-affine equivalent (or have the same integer-affine type), if there exists an affine transformation of $\mathbb{R}^{n+1}$ preserving the set of all integer points and taking the first set to the second. Two sets in $\mathbb{R}^{n+1}$ are said to be integer-linear equivalent (or have the same integer-linear type), if there exists a linear transformation of $\mathbb{R}^{n+1}$ preserving the set of all integer points and taking the first set to the second.

Definition 0.2. A $k$-dimensional plane is called integer if it is integer-affine equivalent to some plane passing through the origin and containing a rank $k$ sublattice of the integer lattice.

Consider some integer $(k-1)$-dimensional plane and an integer point in the complement to this plane. Let the Euclidean distance from the given point to the given plane be equal 1 . The minimal value of nonzero Euclidean distances from all integer points of the ( $k$-dimensional) span of the given plane and the given point to the plane is denoted by $l_{0}$. Note that $l_{0}$ is always greater than zero and can be obtained for some integer point of the described span. The ratio $l / l_{0}$ is said to be the integer distance from the given integer point to the given integer plane.

For example, the integer distance from $O$ to the plane spanned by $A, B$, and $C$ of Fig. 1 equals 3.
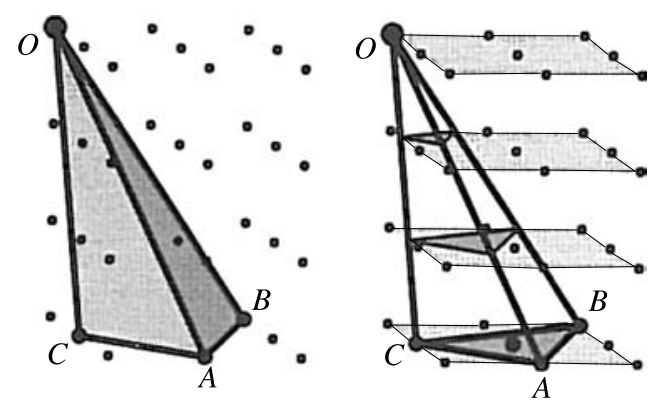

Figure 1. Two images of a completely empty three-story marked pyramid with vertex $O$ and base $A B C$ 
Definition 0.3. An integer convex marked pyramid is called l-story for some positive integer $l$ if the integer distance from the vertex of this pyramid to its base plane equals $l$. An integer convex marked pyramid is called multistory/single-story if the integer distance from the vertex of this pyramid to its base plane is greater than one/equals to one. (See example on Fig. 1.)

For any convex polygon there exists a single-story integer three-dimensional convex marked pyramid with the given polygon as the base (since any single-story integer convex marked pyramid is completely empty). Two single-story threedimensional convex marked pyramids are integer-affine equivalent iff their bases are integer-affine equivalent.

It turns out that the case of multistory convex marked pyramids is essentially different from the single-story case. Only polygons of a few integer-affine types can be bases of multistory convex marked completely empty pyramids. For example, the parallelogram with vertices $(0,0),(0,1),(1,1)$, and $(1,0)$ is not of that type. Besides, there exist integer-affine nonequivalent multistory convex marked completely empty pyramids whose bases are integer-affine equivalent.

In Section 1 of the present paper, we give a complete list of integer-affine types of integer multistory convex marked completely empty pyramids. To classify the pyramids, we study arrangements of integer sublattices on the planes parallel to the bases of the pyramids.

\subsection{Definition of multidimensional continued fractions in the sense of Klein.} The problem of generalizing ordinary continued fractions to the higher-dimensional case was posed by Hermite [9] in 1839. A large number of attempts to solve this problem lead to the birth of several different remarkable theories of multidimensional continued fractions. In this paper we consider the geometrical generalization of ordinary continued fractions to the multidimensional case presented by Klein in 1895 and published by him in [17] and [18].

Consider a set of $n+1$ hyperplanes of $\mathbb{R}^{n+1}$ passing through the origin in general position. The complement to the union of these hyperplanes consists of $2^{n+1}$ open orthants. Let us choose an arbitrary orthant.

Definition 0.4. The boundary of the convex hull of all integer points except the origin in the closure of the orthant is called the sail. The set of all $2^{n+1}$ sails of the space $\mathbb{R}^{n+1}$ is called the $n$-dimensional continued fraction associated to the given $n+1$ hyperplanes in general position in $(n+1)$-dimensional space.

Note that the union of all sails of any continued fraction is centrally symmetric.

In Fig. 2 we show an example of one-dimensional continued fraction. This continued fraction contains four sails (four broken lines in Fig. 2). A description of connections between ordinary continued fractions and geometrical one-dimensional continued fractions can be found in [16], [11], and [12].

Two $n$-dimensional continued fractions are said to be equivalent if there exists a linear transformation that preserves the integer lattice of the $(n+1)$-dimensional space and takes the sails of the first continued fraction to the sails of the other.

Multidimensional continued fractions in the sense of Klein have many relations with other branches of mathematics. For example, Moussafir [27] and 


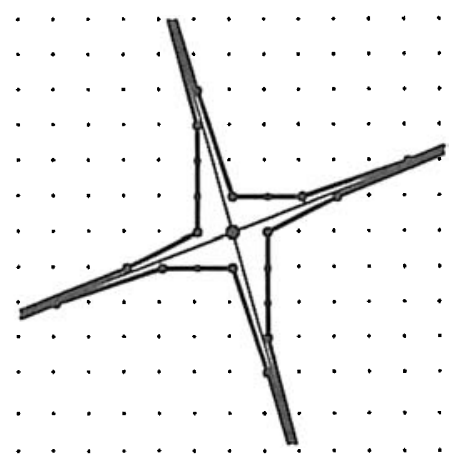

Figure 2. A one-dimensional continued fraction

German [8] studied the connection between the sails of multidimensional continued fractions and Hilbert bases. In [35], Tsuchihashi found the relationship between periodic multidimensional continued fractions and multidimensional cusp singularities, which generalizes the relationship between ordinary continued fractions and two-dimensional cusp singularities. Kontsevich and Suhov discussed the statistical properties of the boundary of a random multidimensional continued fraction in [19]. The combinatorial topological generalization of Lagrange theorem was obtained by Korkina in [21] and its algebraic generalization by Lachaud [24].

The theory of ordinary continued fractions was described, for example, by Hinchin in [10]. Arnold presented a survey of geometrical problems and theorems associated with one-dimensional and multidimensional continued fractions in his articles ([3], [4] and his book [2]). For the algorithms of constructing multidimensional continued fractions, see the papers of Okazaki [30], Moussafir [28].

Korkina in [20], [22], [23] and Lachaud in [24], [25], Bryuno and Parusnikov in [6], [31], and [32], the author in [13] and [14] produced a large number of fundamental domains for periodic algebraic two-dimensional continued fractions. A nice collection of two-dimensional continued fractions is given in the work by Briggs [5].

Besides the multidimensional continued fractions in the sense of Klein, there exist several different generalizations of continued fractions to the multidimensional case. Some other well-known generalizations of continued fractions can be found in the works of Minkowski [29], Voronoi [36], Mittal and Gupta [26], Bryuno and Parusnikov [7], Skorobogat'ko [34], and Shmoilov [33].

0.3. Two-dimensional faces of multidimensional continued fractions. Many classical papers were dedicated to studying algebraic and algorithmic properties of multidimensional continued fractions. The interest to geometrical properties of multidimensional continued fractions was revived by Arnold's work [1] and the subsequent paper of Korkina [20] on the classification of $A$-algebras with three generators. In 1989 and later, Arnold formulated a series of problems and 
conjectures associated to the geometrical and topological properties of sails of multidimensional continued fractions. The majority of these problems are still open. The geometry of sails has not been sufficiently studied.

In the present work, we make the first steps in the investigation of geometric properties of sails. One of the first natural questions here is the following: what compact faces can sails of multidimensional continued fractions have (these objects are usually studied up to the integer-linear equivalence relation)?

The complete answer to this question was known only for one-dimensional continued fractions. For any non-negative integer number $n$ there exists a onedimensional face with exactly $n$ integer points inside. Two compact faces with the same numbers of integer points inside are integer-linear equivalent.

In the two-dimensional case the original question decomposes into two questions.

What compact faces contained in planes at integer distances from the origin equal to one can sails of multidimensional continued fractions have (up to integerlinear equivalence)?

What compact faces contained in planes at integer distances from the origin greater than one can sails of multidimensional continued fractions have (up to integer-linear equivalence)?

The answer to the first question is quite straightforward. For any convex polygon $P$ at the unit integer distance from the origin, there exist a positive integer $k$, a $k$-dimensional continued fraction, and some face $F$ of a sail of this continued fraction, such that $F$ is integer-affine equivalent to $P$. Furthermore, two two-dimensional faces in the planes at the unit integer distance from the origin are integer-linear equivalent iff the corresponding polygons are integer-affine equivalent.

Note that up to this moment the following statement on compact two-dimensional faces (of sails of multidimensional continued fractions) contained in planes at integer distances from the origin greater than one was known. Such faces are either triangles or quadrangles (see the work by Moussafir [3]).

In the present work we classify compact two-dimensional faces contained in planes at integer distances from the origin greater than one up to integer-linear equivalence. This result was announced in [15]. We give complete lists for continued fractions of any dimension. This result is based on the classification of three-dimensional multistory completely empty convex marked pyramids.

0.4. Description of the paper. We start in Section 1 with introducing Theorem A on integer-affine classification of three-dimensional multistory completely empty convex marked pyramids. In this section we also formulate Theorem B on integerlinear classification of two-dimensional faces of the sails at integer distance greater than one. The integer-affine classification of two-dimensional faces contained in planes at integer distances from the origin greater than one (Corollary C) directly follows from the integer-linear classification of two-dimensional faces contained in planes at integer distances from the origin greater than one. In Sections 2 and 3 we prove Theorem A and Theorem B respectively. 

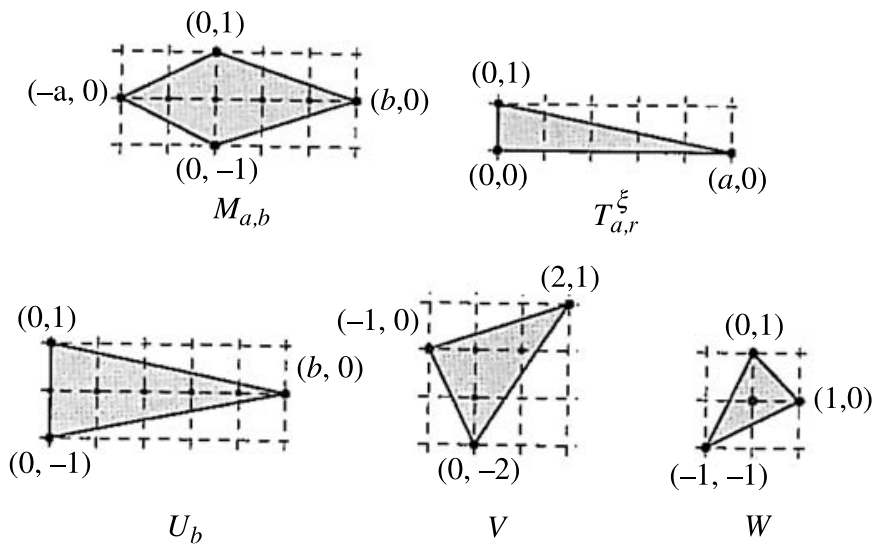

Figure 3. The integer-affine types of the bases of the marked pyramids of list " $\mathrm{M}-\mathrm{W}$ "

\section{Formulation of main results}

\subsection{Classification of two-dimensional multistory completely empty pyra-} mids. By $\left(a_{1}, \ldots, a_{k}\right)$ in $\mathbb{R}^{n}$ for $k<n$ we denote the point $\left(a_{1}, \ldots, a_{k}, 0, \ldots, 0\right)$.

Denote the marked pyramid with vertex at the origin and quadrangular base $(2,-1,0),(2,-a-1,1),(2,-1,2),(2, b-1,1)$, where $b \geqslant a \geqslant 1$, by $M_{a, b}$.

Denote the marked pyramid with vertex at the origin and triangular base

$(\xi, r-1,-r),(a+\xi, r-1,-r),(\xi, r,-r)$, where $a \geqslant 1, r \geqslant 1$, by $T_{a, r}^{\xi}$;

$(2,1, b-1),(2,2,-1),(2,0,-1)$, where $b \geqslant 1$, by $U_{b}$;

$(2,-2,1),(2,-1,-1),(2,1,2)$ by $V$;

$(3,0,2),(3,1,1),(3,2,3)$ by $W$ (the pyramid $W$ is shown on Fig. 1).

The integer-affine types of bases of the described above triangular and quadrangular pyramids are shown on Fig. 3.

Theorem A. Any multistory completely empty convex three-dimensional marked pyramid is integer-affine equivalent exactly to one of the marked pyramids from the following list.

List "M-W":

- the quadrangular marked pyramids $M_{a, b}$, with integers $b \geqslant a \geqslant 1$;

- the triangular marked pyramids $T_{a, r}^{\xi}$, where $a \geqslant 1$, and $\xi$ and $r$ are relatively prime, and $r \geqslant 2$ and $0<\xi \leqslant r / 2$;

- the triangular marked pyramids $U_{b}$, where $b \geqslant 1$;

- the triangular marked pyramid $V$;

- the triangular marked pyramid $W$.

We give the proof of Theorem A in Section 2.

1.2. Compact two-dimensional faces at distance greater than one. Note that the following statement on compact two-dimensional faces contained in planes at the integer distance from the origin greater than one was known. 
Theorem (Moussafir [28]). Let $F$ be a two-dimensional compact face of some sail of a two-dimensional continued fraction. Let $r$ be the integer distance from the origin to the plane, containing the face.

1. If $r=1$, then $F$ may have arbitrary many vertices.

2. If $r=2$, then $F$ has at most 4 vertices.

3. If $r \geqslant 3$, then $F$ has three vertices.

Here we present a new theorem on integer-linear classification and its corollary on integer-affine classification of two-dimensional faces of multidimensional sails (the faces are again contained in the planes at integer distances greater than one from the origin). Note that from this theorem and its corollary it follows that the second item of Moussafir's theorem can be strengthened:

$2^{\prime}$. If $r=2$, then $F$ has three vertices.

Quadrangular faces for the case of $r=2$ are possible only for $n$-dimensional continued fractions where $n \geqslant 3$.

Theorem B. Any compact two-dimensional face of a sail of a two-dimensional continued fraction contained in a plane at integer distance from the origin greater than one is integer-linear equivalent exactly to one of the faces of the following list.

List " $\alpha_{2}$ ":

- triangle with vertices $(\xi, r-1,-r),(a+\xi, r-1,-r),(\xi, r,-r)$, where $a \geqslant 1$, integers $\xi$ and $r$ are relatively prime and satisfy the following inequalities $r \geqslant 2$ and $0<\xi \leqslant r / 2$;

- triangle with vertices $(2,1, b-1),(2,2,-1)$, and $(2,0,-1)$ for $b \geqslant 1$;

- triangle with vertices $(2,-2,1),(2,-1,-1)$, and $(2,1,2)$;

- triangle with vertices $(3,0,2),(3,1,1)$, and $(3,2,3)$.

All triangular faces of list " $\alpha_{2}$ " are realizable by sails of dimension two and integer-linear nonequivalent to each other.

Any compact two-dimensional face of a sail of an n-dimensional $(n \geqslant 3)$ continued fraction contained in a plane at integer distance from the origin greater than one is integer-linear equivalent exactly to one of the faces of the following list.

List " $\alpha_{\mathrm{n}}$ ", $n \geqslant 3$ :

- quadrangle with vertices $(2,-1,0),(2,-a-1,1),(2,-1,2),(2, b-1,1)$, for $b \geqslant a \geqslant 1$

- triangle with vertices $(\xi, r-1,-r),(a+\xi, r-1,-r),(\xi, r,-r)$, where $a \geqslant 1$, integers $\xi$ and $r$ are relatively prime and satisfy the following inequalities $r \geqslant 2$ and $0<\xi \leqslant r / 2$;

- triangle with vertices $(2,1, b-1),(2,2,-1)$, and $(2,0,-1)$ for $b \geqslant 1$;

- triangle with vertices $(2,-2,1),(2,1,2)$, and $(2,-1,-1)$;

- triangle with vertices $(3,0,2),(3,1,1)$, and $(3,2,3)$.

All faces of list " $\alpha_{n}$ " are realizable by sails of any dimension greater than two and integer-linear nonequivalent to each other. 
Remark 1.1. Note that for any compact face of a sail we can associate an integer completely empty convex marked pyramid with marked vertex at the origin and this face as base. Therefore integer-affine types of such marked pyramids are in one-to-one correspondence with integer-linear types of faces (see Lemma 3.1 below).

We give a proof of Theorem B in Section 3.

Corollary C. Any compact two-dimensional face of a sail of a multidimensional continued fraction contained in a plane at integer distance from the origin equals $r$ is integer-affine equivalent exactly to one of the polygons of the list $\beta_{r}$ shown below.

List " $\beta_{2}$ ":

- quadrangle with vertices $(-1,0),(-a-1,1),(-1,2),(b-1,1)$, where $b \geqslant a \geqslant 1$ (see the case of $a=2, b=3$ on Fig. 4a); quadrangular faces are possible only for $n$-dimensional continued fractions where $n \geqslant 3$;

- single triangle $(-1,0),(0,-2),(2,1)$ (see Fig. 4b);

- triangle $(0,-1),(0,1),(b, 0)$, for $b \geqslant 1$ (see the case of $b=5$ on Fig. $4 c)$;

- triangle $(0,0),(a, 0),(0,1)$, for $a \geqslant 1$ (see the case of $a=5$ on Fig. $4 d$ ).

List " $\beta_{3}$ ":

- single triangle $(-1,-1),(1,0),(0,1)$ (see Fig. 5a);

- triangle $(0,0),(a, 0),(0,1)$, for $a \geqslant 1$ (see the case of $a=5$ on Fig. $5 b$ ).

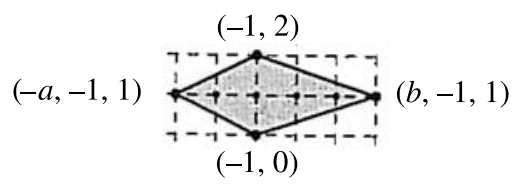

a)

$(2,1)$

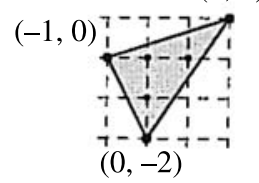

b)

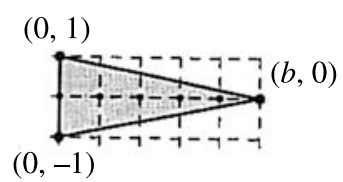

c)

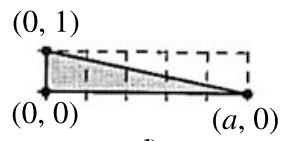

d)

Figure 4. Integer-affine types of faces of list " $\beta_{2}$ ”

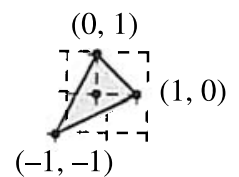

a)

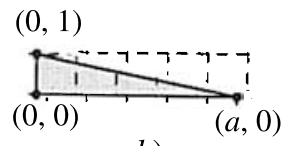

b)

Figure 5. Integer-affine types of faces of list " $\beta_{3}$ ", 


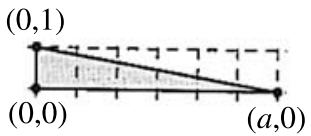

Figure 6. Integer-affine types of faces of list " $\beta_{r}$ ", for $r \geqslant 4$

$$
\text { List " } \beta_{r} \text { " }(r \geqslant 3) \text { : }
$$

- triangle with vertices $(0,0),(a, 0)$, and $(0,1)$, for some $a \geqslant 1$ (see the case of $a=6$ on Fig. 6), the corresponding convex marked pyramid is integer-affine equivalent to $T_{a, r}^{\xi}$, where the integers $\xi$ and $r$ are relatively prime and satisfy $0<\xi \leqslant r / 2$. For different $\xi$ the corresponding faces are integer-linear nonequivalent but integer-affine equivalent.

For any integer $r$ the faces of list $\beta_{r}$ are integer-affine nonequivalent to each other; list $\beta_{r}$ is irredundant.

The integer-affine and the integer-linear classifications coincide, for $r<5$. For $r \geqslant 5$, the integer-linear classification contains the integer-affine classification.

For any integers $n \geqslant 3$ and $r \geqslant 2$, the integer-linear classification of compact two-dimensional faces contained in planes at integer distances from the origin greater than one of sails of $n$-dimensional continued fractions coincides with the integer-affine classification of completely empty $r$-story three-dimensional convex marked pyramids.

\section{Proof of Theorem A}

2.1. Preliminary definitions and statements. Let us give several definitions, fix the notation, and also formulate some general statements that we will further use in the proofs.

For an integer polygon in some two-dimensional subspace the ratio of its Euclidean volume to the minimal possible Euclidean volume of an integer triangle in the same two-dimensional subspace is called the integer volume of this polygon.

An integer polyhedron (polygon) is called empty, if it does not contain integer points in its interior, and the set of integer points of the faces coincides with the set of vertices of the polyhedron (polygon).

Let $A B C D$ be a tetrahedron with an ordered set of vertices $A, B, C$, and $D$. Denote by $P(A B C D)$ the following parallelepiped:

$$
\{A+\alpha \overline{A B}+\beta \overline{A C}+\gamma \overline{A D} \mid 0 \leqslant \alpha \leqslant 1,0 \leqslant \beta \leqslant 1,0 \leqslant \gamma \leqslant 1\} .
$$

Definition 2.1. Now we specify some useful coordinates (denoted by $\langle x, y, z\rangle$ ) in the three-dimensional subspace containing $P(A B C D)$ of $\mathbb{R}^{n}$. Let $b, c$, and $d$ be the integer distances from $B, C$, and $D$ to the two-dimensional planes containing the faces $A C D, A B D$, and $A C D$ respectively. Let us define the coordinates of $A, B, C$, and $D$ as follows: $\langle 0,0,0\rangle,\langle b, 0,0\rangle,\langle 0, c, 0\rangle$, and $\langle 0,0, d\rangle$ respectively. 
The coordinates of all other points in this three-dimensional subspace are uniquely defined by means of linearity. We call them the integer-distance coordinates with respect to $P(A B C D)$.

Remark 2.2. For any set of vertices $A, B, C$, and $D$ ordered as in $P(A B C D)$, the integer-distance coordinates are uniquely defined.

By integer lattice nodes of $\mathbb{R}^{n}$ (or, for short, lattice nodes) we mean integer points in the original coordinates in $\mathbb{R}^{n}$.

Remark 2.3. Note that any lattice node of the three-dimensional space described above has integer coordinates in the new integer-distance system of coordinates. The inverse is not true. There exist an integer-distance system of coordinates and a point in the corresponding three-dimensional space with integer coordinates which is not a lattice node. For lattice nodes, the absolute values of their new coordinates coincide with integer distances from these lattice nodes to the planes containing the corresponding faces of the parallelepiped.

Let us continue with the following definition.

Definition 2.4. Two points $P$ and $Q$ are said to be equivalent with respect to some integer parallelogram $A B C D$ if there exist integers $\lambda$ and $\beta$ such that $P=Q+\lambda \overline{A B}+\beta \overline{A C}$. The set of all equivalence classes of the integer lattice with respect to the integer parallelogram $A B C D$ is called the quotient-lattice of the space by this integer parallelogram.

Note that any equivalence class is contained in one of the two-dimensional planes parallel to the plane of the parallelogram.

Proposition 2.5. Consider an integer parallelepiped $A B C D A^{\prime} B^{\prime} C^{\prime} D^{\prime}$ in $\mathbb{R}^{3}$ and some integer plane $\pi$ parallel to the face $A B C D$. Let $\pi$ intersect the parallelepiped (along a parallelogram). Then the following two statements hold.

First, $\pi$ contains only finitely many equivalence classes of the integer lattice with respect to the integer parallelogram $A B C D$. Their number equals to the index of the sublattice generated by the vectors $\overline{A B}$ and $\overline{A C}$ in the integer lattice of the plane containing $A B C D$.

Second, for any equivalence class of the integer lattice contained in $\pi$ with respect to the integer parallelogram $A B C D$ it holds exactly one of the following conditions.

a) Only one point of the equivalence class is in the parallelogram, it is an interior point of the parallelogram;

b) two points of the equivalence class are in the parallelogram, they are contained in opposite (open) edges of the parallelogram;

c) four points of the equivalence class are in the parallelogram, they coincide with vertices of the parallelogram.

We skip the proof of Proposition 2.5. It is straightforward and is based on the following easy lemma. 
Lemma 2.6. Consider an integer parallelepiped with an empty face. Let some parallel to this face plane intersect the parallelepiped. Then exactly one of the following statements holds.

a) Only one lattice node is in the parallelogram, it is an interior point;

b) two lattice nodes are in the parallelogram, they are contained in (open) opposite edges of the parallelogram;

c) four lattice nodes are in the parallelogram, they coincide with vertices of the parallelogram.

Further we use the following corollary of Proposition 2.5.

Corollary 2.7. Consider an integer parallelepiped $A B C D A^{\prime} B^{\prime} C^{\prime} D^{\prime}$ in $\mathbb{R}^{3}$. Denote by $d$ the integer distance between $A^{\prime}$ and $B A D$. Denote by $s$ the number of equivalence classes of the integer lattice with respect to the integer parallelogram $A B C D$ that are contained in the plane of $A B C D$. Finally, denote by $v$ the number of equivalence classes of the integer lattice with respect to the parallelogram $A B C D$ that are contained either strictly between two planes of faces $A B C D$ and $A^{\prime} B^{\prime} C^{\prime} D^{\prime}$ or in the plane of $A B C D$. Then we have

$$
d=\frac{v}{s} .
$$

Proof. It follows from Proposition 2.5 that each integer plane parallel to $A B C D$ contains exactly $s$ equivalence classes. Hence there are exactly $v / s-1$ integer planes between two planes containing faces $A B C D$ and $A^{\prime} B^{\prime} C^{\prime} D^{\prime}$. Therefore, $d=v / s$.

2.2. First results on empty integer tetrahedra. In this subsection we show the corollary of White's theorem (see also [8]). Here without loss of generality we consider only the three-dimensional space. The result of White [37] implies, as a special case, the following theorem.

Theorem 2.8 (White [37]). Let $\Delta \subset \mathbb{R}^{3}$ be an integer three-dimensional simplex, let $E_{i}=\left\{\sigma_{i}, \sigma_{i}^{\prime}\right\}, i=1,2,3$ be the set of points belonging to a pair of opposite edges $\sigma_{i}, \sigma_{i}^{\prime}$ of $\Delta$. Then $\left(\Delta \backslash E_{i}\right) \cap \mathbb{Z}^{3}$ is empty iff there exist $j \in\{1,2,3\}$ and two neighboring planes $\pi_{j}, \pi_{j}^{\prime}$ (by neighbor we mean that there are no integer lattice nodes "between" these planes $\pi_{j}$ and $\pi_{j}^{\prime}$, such that $\sigma_{i} \subset \pi_{j}$ and $\sigma_{i}^{\prime} \subset \pi_{j}^{\prime}$.

We will use the following corollary on empty integer tetrahedra for the classification of empty convex multistory tetrahedra and also further in the proof of Theorem A.

Corollary 2.9. Let $A D B A^{\prime}$ be some empty integer tetrahedron. Then all integer interior lattice nodes of the parallelepiped $P\left(A D B A^{\prime}\right)$ are in the plane passing through two centrally-symmetric edges of the parallelepiped. These two edges do not contain the vertex $A$.

Proof. Consider an empty integer tetrahedron $A D B A^{\prime}$ and the corresponding parallelepiped $P\left(A D B A^{\prime}\right)=A B C D A^{\prime} B^{\prime} C^{\prime} D^{\prime}$. Without loose of generality we suppose that the statement of Theorem 2.9 holds for the edges $A A^{\prime}$ and $B D$ of the 
tetrahedron $A D B A^{\prime}$. We obtain that there are no lattice nodes between the plane $\pi_{1}$ containing the central section $B B^{\prime} D^{\prime} D$ and $\pi_{2}$ parallel to $\pi_{1}$ and passing through the segment $A A^{\prime}$. So all lattice nodes of the prism $A B D A^{\prime} B^{\prime} D^{\prime}$ distinct to the points $A$ and $A^{\prime}$ are contained in $\pi_{1}$ (i.e. in $B B^{\prime} D^{\prime} D$ ).

Note that both points $P$ and $P^{\prime}=A+\overline{P C^{\prime}}$ are at the same time lattice nodes or not, since $A$ and $C^{\prime}$ are lattice nodes. If $P$ is in the prism $C B D C^{\prime} B^{\prime} D^{\prime}$ then $P^{\prime}$ is in $A B D A^{\prime} B^{\prime} D^{\prime}$. Therefore all lattice nodes of the prism $A B D A^{\prime} B^{\prime} D^{\prime}$ distinct to the points $C$ and $C^{\prime}$ are also contained in $\pi_{1}$ (i.e. in $B B^{\prime} D^{\prime} D$ ). This concludes the proof of the corollary.

Remark 2.10. The number of planes passing through two centrally-symmetric edges of the parallelepiped equals six, and only three of them do not contain the vertex $A$.

2.2.1. Classification of empty triangular marked pyramids. Corollary 2.9 allows to describe all integer-affine types of empty triangular marked pyramids (i.e. tetrahedra with one marked vertex each).

Let $r$ be some positive integer, and $\xi$ be a nonnegative integer. Denote by $P_{r}^{\xi}$ the marked pyramid with vertex at $(0,0,0)$ and triangular base $(0,1,0),(1,0,0)$, $(\xi, r-\xi, r)$.

Corollary 2.11. Any integer empty triangular marked pyramid is integer-affine equivalent to exactly one of the pyramids of

List " $P$ ":

$-P_{1}^{0}$;

- $P_{r}^{\xi}$, where $\xi$ and $r$ are relatively prime, $r \geqslant 2$, and $0<\xi \leqslant r / 2$.

All triangular marked pyramids of list " $P$ " are empty and integer-affine nonequivalent to each other.

Proof. 1. Completeness of list " $P$ ". Let us show that an arbitrary empty integer marked pyramid $A D B A^{\prime}$ (with a vertex $A$ ) is integer-affine equivalent to one of the marked pyramids of " $P$ ".

Suppose that the integer distance from its marked vertex to the plane containing the marked base equals some positive integer $r$. If $r=1$ then the vertices of the marked pyramid generate the three-dimensional integer lattice, and therefore such a marked pyramid is integer-affine equivalent to $P_{1}^{0}$ (here $A$ corresponds to the marked vertex of $P_{1}^{0}$ ).

Suppose now that $r>1$. By Corollary 2.9 all lattice nodes of the parallelepiped $P\left(A D B A^{\prime}\right)$ are contained exactly in one of the three planes passing through centrally-symmetric edges of the parallelepiped and not containing $A$. Denote the vertices of the marked base $D B A^{\prime}$ by $\bar{B}, \bar{D}$, and $\bar{A}^{\prime}$ in such a way that all interior lattice nodes of the parallelepiped $P\left(A \overline{D B A}^{\prime}\right)$ are contained in the plane passing through $\overline{B D}$ and the centrally-symmetric edge.

Consider the integer-distance coordinates with respect to the parallelepiped $P\left(A \overline{D B A}^{\prime}\right)$. By Corollary 2.7 the coordinates of $A^{\prime}, B$, and $D$ equal to $\langle r, 0,0\rangle$, $\langle 0, r, 0\rangle$, and $\langle 0,0, r\rangle$ respectively. Take the intersection of the parallelepiped with 
the plane $x=1$ in these coordinates. There is only one lattice node in the intersection, by Corollary 2.9 its coordinates are $\langle 1, r-\xi, \xi\rangle$. Denote this lattice node by $K$.

If the integers $\xi$ and $r$ have some common integer divisor $c \geqslant 1$, then the point with the coordinates $\left\langle\frac{r}{c}, \frac{r-\xi}{c} r, \frac{\xi}{c} r\right\rangle$ is a lattice node. Hence the point $\langle r / c, 0,0\rangle$ is also a lattice node. The marked pyramid $A \overline{D B A}^{\prime}$ is not empty, since it contains $\langle r / c, 0,0\rangle$. Thus the integers $\xi$ and $r$ are relatively prime.

Since the integer distance from $K$ to the two-dimensional plane containing the face $A \overline{D B}$ equals one, there exists an integer-affine transformation taking the tetrahedron $A \overline{B D} K$ to the tetrahedron with vertices $(0,0,0),(0,1,0),(1,0,0)$, and $(1,1,1)$. Here the point $\bar{A}^{\prime}$ maps to $(\xi, r-\xi, r)$. Hence the integer-affine type of the marked pyramid $A B D A^{\prime}$ coincides with the integer-affine type of the marked pyramid $A \overline{B D A}$, and therefore it coincides with the integer-affine type of the marked pyramid $P_{r}^{\xi}$, where $0<\xi<r$, and $\xi$ and $r$ are relatively prime. It remains to say that the marked pyramids $P_{r}^{\xi}$ and $P_{r}^{r-\xi}$ can be mapped one to another by the integer-affine symmetry preserving the points $(0,0,0),(0,0,1)$, and $(1,1,0)$, and transposing $(1,0,0)$ and $(0,1,0)$. Therefore the marked pyramids $P_{r}^{\xi}$ and $P_{r}^{r-\xi}$ are integer-affine equivalent.

2. Emptiness of the marked pyramids of list "P". Let us show that all listed marked pyramids $P_{\xi}^{r}$ are empty. The intersection of the plane $a_{3}=b$ (for $1 \leqslant b \leqslant(r-1))$ and marked pyramid $P_{\xi}^{r}$ is the triangle $A_{k} B_{k} D_{k}$ with the following coordinates of the vertices:

$$
\left(\frac{b}{r} \xi, \frac{b}{r}(r-\xi), b\right), \quad\left(\frac{b}{r}, \frac{b}{r}(r-\xi)+\frac{r-b}{r}, b\right), \quad\left(\frac{b}{r} \xi+\frac{r-b}{r}, \frac{b}{r}(r-a), b\right) .
$$

The triangle $A_{k} B_{k} D_{k}$ is contained in the band $b \leqslant a_{1}+a_{2} \leqslant b+\frac{r-b}{r}, a_{3}=b$. This band contains only integer points with coordinates $(t, b-t, b)$ for integer $t$. Hence it remains to check if $A_{k}$ is integer. Since $\xi$ and $r$ are relatively prime and $d<r$, the first coordinate of $A_{k}$ is not integer. Therefore all marked pyramids $P_{\xi}^{r}$ of list " $P$ " are empty.

3. Irredundance of list " $P$ ". We will show now that all marked pyramids $P_{\xi}^{r}$ of list " $P$ " are integer-affine nonequivalent to each other. Note that the integer distance from the marked vertex to the plane containing the base is an integeraffine invariant. Therefore the pyramids with distinct parameter $r$ are integer-affine nonequivalent.

To distinguish the marked pyramids with the same $r$, we construct the following integer-affine invariant. Consider an arbitrary empty marked pyramid $A B D A^{\prime}$ with marked vertex $A$ and the corresponding trihedral angle also with vertex $A$ and triangle $D B A^{\prime}$ as its base. By White's theorem exactly one lattice node of the trihedral angle (we denote this lattice node by $K$ ) is contained in the twodimensional plane parallel to the face $D B A^{\prime}$ and at integer distance $r+1$ from $A$. By Corollary 2.9 the integer distances from $K$ to two-dimensional planes of the angle equal $1, \xi, r-\xi$ (for some integer $\xi$ ). The trihedral angle and $K$ are uniquely defined by the marked pyramid up to the symmetries of the marked pyramid preserving the marked vertex. The group of such symmetries permutes all integer 
distances from $K$ to the planes containing the faces of the angle. Hence, the unordered system of integers $[1, \xi, r-\xi]$ is an invariant. This invariant distinguishes all marked pyramids $P_{\xi}^{r}$ with the same integer distance $r$.

Proposition 2.12. Let relatively prime integers $\xi$ and $r$ satisfy the following inequalities: $r \geqslant 2,0<\xi \leqslant r / 2$. Then the marked pyramid $P_{r}^{\xi}$ is integer-affine equivalent to the marked pyramid $T_{1, r}^{\xi}$.

Proof. The marked pyramid $T_{1, r}^{\xi}$ is the image of $P_{r}^{\xi}$ under the integer-linear transformation

$$
\left(\begin{array}{ccc}
\xi+1 & \xi & -\xi \\
r-1 & r-1 & 2-r \\
-r & -r & r-1
\end{array}\right)
$$

Corollary 2.13. Any integer empty $r$-story $(r \geqslant 2)$ triangular marked pyramid is integer-affine equivalent exactly to one of the marked pyramids $T_{1, r}^{\xi}$ for relatively prime integers $\xi$ and $r$ satisfying $0<\xi \leqslant r / 2$. All such pyramids $T_{1, r}^{\xi}$ are empty (and integer-affine nonequivalent if the corresponding parameters $r$ and $\xi$ do not coincide).

2.2.2. Classification of integer empty tetrahedra. A certain difference between the integer-affine classification of integer empty triangular marked pyramids (with marked vertex) and the integer-affine classification of integer empty tetrahedra (without marked vertices) occurs. The first steps in the integer-affine classifications of integer empty tetrahedra were made by Moussafir in [28].

Theorem 2.14 (Moussafir [28]). Any integer empty tetrahedron is integer-affine equivalent to the tetrahedron with vertices $(0,0,0),(1,0,0),(0,1,0)$, and $(u, v, d)$, for some integers $u, v$ and $d$, where $u, v$ and $u+v-1$ are relatively prime with $d$, and one of the integers $u+v, u-1, v-1$ is divisible by $d$. (These tetrahedra are sometimes called Hermitian normal forms of the simplices.)

Note that many of such Hermitian normal forms are integer-affine equivalent to each other. The following consequence of Corollary 2.9 improves Moussafir's theorem.

Corollary 2.15. Any integer empty tetrahedron is integer-affine equivalent exactly to one of the following tetrahedra:

$-P_{1}^{0}$

- $P_{r}^{\xi}$, where $r \geqslant 2,0<\xi<r$, and the element $(\xi \bmod r$ ) of the additive group $\mathbb{Z} / m \mathbb{Z}$ is also contained in the associated multiplicative group $(\mathbb{Z} / m \mathbb{Z})^{*}$ (i.e. integers $\xi$ and $r$ are relatively prime).

All listed integer tetrahedra are empty. Two tetrahedra $P_{r_{1}}^{\xi}$ and $P_{r_{2}}^{\nu}$ are integeraffine equivalent iff $r_{1}=r_{2}$ and (for $r_{1}>1$ ) one of the following equalities in $(\mathbb{Z} / m \mathbb{Z})^{*}$ holds:

$$
\left(\xi \bmod r_{1}\right)=( \pm 1) \cdot\left(\nu \bmod r_{1}\right)^{ \pm 1}
$$


Proof. 1. Completeness of the list. By Corollary 2.11 any empty integer tetrahedron is integer-affine equivalent to some tetrahedron of the list of Corollary 2.15.

2. Emptiness of the tetrahedra of the list. By Corollary 2.11 the tetrahedron $P_{r}^{\xi}$ is empty for relatively prime integers $r$ and $\xi$ satisfying $r \geqslant 2$ and $\xi \leqslant r / 2$. Since $P_{r}^{\xi}$ and $P_{r}^{r-\xi}$ are integer-affine equivalent and $P_{1}^{0}$ is empty, all tetrahedra of the list of Corollary 2.15 are empty.

3. Proof of the last statement of Corollary 2.15. Consider any tetrahedron $P_{r}^{\xi}$ of the list. The set of four trihedral angles associated with all four vertices of the tetrahedron is uniquely defined.

It follows from White's theorem, that for any of these trihedral angles exactly one lattice node contained in the interior of the angle is at unit integer distance to the face of tetrahedron do not containing the vertex of the angle. Direct calculations show that the integer distances from these points to the four two-dimensional planes containing the faces of the tetrahedron are

$$
(1,1, \xi, r-\xi), \quad(1,1, \xi, r-\xi), \quad(\nu, r-\nu, 1,1), \quad \text { and } \quad(\nu, r-\nu, 1,1),
$$

where $(\xi \bmod r) \cdot(\nu \bmod r)=1$ in $(\mathbb{Z} / m \mathbb{Z})^{*}$. The set of these numbers up to the group $S_{4}$ of permutations action (for all points at the same time) is an integer-affine invariant. Therefore, the tetrahedra $P_{r}^{\xi}, P_{r}^{\nu}, P_{r}^{r-\xi}$, and $P_{r}^{r-\nu}$ are integer-affine equivalent and the invariant distinguishes all other tetrahedra.

Remark 2.16. The integer-affine classifications of integer empty triangular marked pyramids and of integer empty tetrahedra coincide only for $r=1,2,3$, $4,5,6,8,10,12,24$.

2.3. Proof of Theorem A for the case of polygonal marked pyramids. In this subsection we study the case of marked pyramids with polygonal bases (containing more than three angles distinct from the straight angle). In the next subsection we will study triangular marked pyramids.

\subsubsection{Integer parallelograms contained in integer polyhedra.}

Proposition 2.17. Let four vertices of a convex polygon be integer points. Then this polygon contains some integer parallelogram that is integer-affine equivalent either to the unit parallelogram, or to the parallelogram with vertices $(1,0),(0,1)$, $(-1,0)$, and $(0,-1)$.

Proof. Suppose that a convex polygon contains four integer vertices, denote them by $K, L, M$, and $N$. Let us show that the quadrangle $K L M N$ contains some integer parallelogram.

Define $M^{\prime}=N+\overline{K L}$. The vertex $M$ can be in any of the four orthants with respect to the lines containing $M^{\prime} N$ and $M^{\prime} L$. For any of these four cases, we explicitly construct an integer parallelogram contained in the quadrangle on Fig. 7 (we draw the quadrangle $K L M N$ with thick line, the corresponding parallelogram is shaded).

Let some point of an integer parallelogram be integer. Consider the point which is centrally-symmetric about the intersection point of the diagonals of this parallelogram. This point is also in the parallelogram and is integer. 

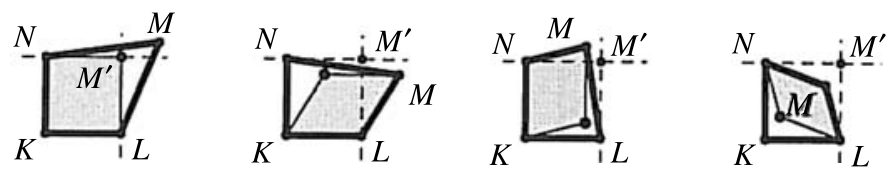

Figure 7. Possible cases for $M^{\prime}$ with respect to the quadrangle $K L M N$

Denote the integer parallelogram in the polygon by $A B C D$.

1. Integer empty parallelogram. Suppose $A B C D$ is empty. Then it generates the integer lattice and hence is integer-affine equivalent to the standard one.

2. Integer parallelogram with the only one integer point inside. Suppose $A B C D$ contains only one integer point $O$ in its interior. Then this point coincides with the centrally-symmetric point about the intersection point of the diagonals of this parallelogram. And hence it coincides with the intersection point of the diagonals. Therefore the integer triangle $O A B$ is empty. Hence it is integer-affine equivalent to the standard unit triangle. Thus $A B C D$ is integer-affine equivalent to the parallelogram with vertices $(1,0),(0,1),(-1,0)$, and $(0,-1)$.

3. Remaining cases. Suppose that the parallelogram $A B C D$ contains more than one integer point except of the vertices. Then there exists a point among these points such that it is distinct to the intersection point of the diagonals of this parallelogram. We denote it by $O$. Denote the centrally-symmetric point about the intersection point of the diagonals of this parallelogram by $O^{\prime}$. Without loss of generality, we suppose that $O O^{\prime}$ is not a subset of $A C$ (otherwise $O O^{\prime}$ is not a subset of $B D$ ). Therefore $A O C O^{\prime}$ (or $A O^{\prime} C O$ ) is an integer parallelogram contained in $A B C D$. The number of integer points of $A O C O^{\prime}$ is smaller than the number of integer points of $A B C D$ at least by two. Hence we come to one of the cases of item 1. or 2. in a finite number of such steps.

Therefore any convex polygon with four integer vertices contains a parallelogram integer-affine equivalent to one of the parallelograms of Proposition 2.17.

\subsubsection{The case of an empty marked pyramid with an empty parallelogram as base.}

Proposition 2.18. Let an empty integer parallelogram be a base of some marked pyramid. If this pyramid is empty, then it is single-story.

Proof. We prove by reductio ad absurdum. Let $A^{\prime} A B C D$ be an empty marked pyramid with marked vertex $A^{\prime}$ and an empty parallelogram $A B C D$ as its base. Suppose that the integer distance from the point $A^{\prime}$ to the plane containing $A B C D$ equals $r>1$. Consider the parallelepiped $P\left(A A^{\prime} B C\right)$ and the integer-distance coordinates corresponding to it (denoted by $\langle x, y, z\rangle$ ). By Corollary 2.7 the coordinates of $A^{\prime}, B$, and $C$ equal to $\langle r, 0,0\rangle,\langle 0, r, 0\rangle$, and $\langle 0,0, r\rangle$ respectively. Note that coordinates of lattice nodes (in old coordinates) are integers.

Let us find the lattice node of the parallelepiped at unit integer distance to the plane containing $A B C$, i.e. the lattice node with coordinates $\langle 1, y, z\rangle$, where $0 \leqslant y \leqslant r, 0 \leqslant z \leqslant r$. On the one hand, it is not contained in the marked pyramid $A^{\prime} A B C D$, and hence $y+1>r$ or $z+1>r$. On the other hand, by Corollary 2.9 the two-dimensional faces of $P\left(A A^{\prime} B C\right)$ do not contain integer points distinct to 
vertices, since $A A^{\prime} B C$ is empty. Therefore $y \neq r$ and $z \neq r$. Hence there are no lattice nodes in the plane containing $A B C$. We come to the contradiction with Lemma 2.6.

\subsubsection{The case of a completely empty marked pyramid whose base is an integer parallelogram containing a unique integer point in its interior.}

Lemma 2.19. Consider an integer marked pyramid with vertex $O$ and parallelogram $A B C D$ as base. Let $A B C D$ be integer-affine equivalent to the parallelogram with vertices $(1,0),(0,1),(-1,0)$, and $(0,-1)$. If the marked pyramid $O A B C D$ is completely empty and multistory, then it is two-story. The integer-affine type of such a pyramid coincides with the integer-affine type of the pyramid with vertex $(0,0,0)$ and base $(2,-1,0),(2,-2,1),(2,-1,2),(2,0,1)$.

Proof. Let the integer base $A B C D$ of the completely empty $r$-story integer marked pyramid $O A B C D(r \geqslant 2)$ be integer-affine equivalent to the parallelogram with vertices $(1,0),(0,1),(-1,0)$, and $(0,-1)$.

Consider the parallelepiped $P(A O B C)$ and the integer-distance coordinates corresponding to it (denoted by $\langle x, y, z\rangle$ ). By Corollary 2.7 the coordinates of $O$, $B, C$, and $D$ equal $\langle r, 0,0\rangle,\langle 0,2 r, 0\rangle,\langle 0,0,2 r\rangle$, and $\langle 0,2 r, 2 r\rangle$ respectively.

Let us consider the parallelogram of intersection of $P(A O B C)$ with the plane $x=1$. Now we find all lattice nodes in this parallelogram. By Proposition 2.5 there are exactly two lattice nodes in the parallelogram of intersection. Let us describe all possible positions of these nodes in the intersection of $P(A O B C)$ and the plane $x=1$. First, there are no lattice nodes in the intersection of the marked pyramid $A O B C D$ and the plane $x=1$, i.e. in the closed parallelogram with vertices $\langle 1,0,0\rangle,\langle 1,0,2 r-2\rangle,\langle 1,2 r-2,2 r-2\rangle$, and $\langle 1,2 r-2,0\rangle$. Secondly, there are no lattice nodes in all parallelograms obtained from the given one by applying translations by the vectors $\lambda\langle 0,2 r, 0\rangle+\mu\langle 0, r, r\rangle$, where $\lambda$ and $\mu$ are integers. On Fig. 8, we show some parallelograms that do not contain any lattice nodes. These parallelograms are painted shaded.

So, the lattice nodes of the intersection parallelogram of $P(A O B C)$ with the plane $x=1$ can only coincide with integer points of open parallelograms obtained from the parallelogram with vertices $K\langle 1, r-2,2 r-2\rangle, L\langle 1, r, 2 r-2\rangle, M\langle 1, r, 2 r\rangle$, and $N\langle 1, r-2,2 r\rangle$ by the symmetry with respect to the plane $y=z$ and translations by the vectors $\lambda\langle 0,2 r, 0\rangle+\mu\langle 0, r, r\rangle$, where $\lambda$ and $\mu$ are integers. The parallelogram $K L M N$ contains exactly one integer point $\langle 1, r-1,2 r-1\rangle$, see Fig. 8 .

Suppose that this point is a lattice node. Since the intersection parallelogram contains exactly two lattice nodes, the point symmetric to the point $\langle 1, r-1,2 r-1\rangle$ with respect to the plane $y=z$ is also a lattice node (there are no other integer points in the intersection parallelogram). Therefore $\langle 2,2 r-2,4 r-2\rangle$ is a lattice node. Hence $\langle 2,2 r-2,2 r-2\rangle$ is a lattice node, and hence $\langle 2, r-2, r-2\rangle$ is also a lattice node. However, for $r \geqslant 3$ the point $\langle 2, r-2, r-2\rangle$ is contained in the closed parallelogram of intersection of $P(A O B C)$ with the plane $x=2$. The vertices of this parallelogram are the following: $\langle 2,0,0\rangle,\langle 1,0,2 r-4\rangle$, $\langle 1,2 r-4,2 r-4\rangle$, and $\langle 1,2 r-4,0\rangle$. Thus there are no pyramids satisfying all the conditions of Lemma 2.19 for $r \geqslant 3$. 


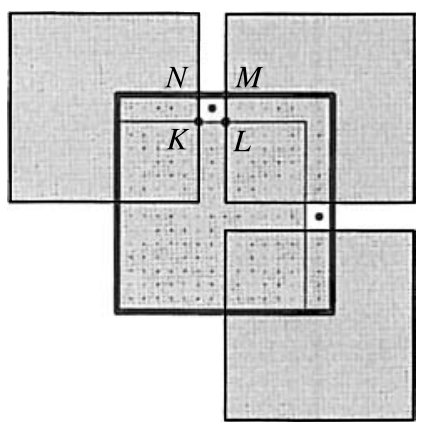

Figure 8. The intersection of $P(A O B C)$ and the plane $x=1$

Now consider the case $r=2$. The integer points $A, B, C$, and $\langle 1,1,3\rangle$ define the integer lattice in a unique way. This implies that all marked pyramids satisfying all the conditions of Lemma 2.19 are of the same integer-affine type, and it coincides with the integer-affine type of the marked pyramid with vertex $(0,0,0)$ and base $(2,-1,0),(2,-2,1),(2,-1,2),(2,0,1)$ (in the old coordinates).

2.3.4. General case. Now we study the general case of integer completely empty marked pyramids with convex polygonal bases.

Lemma 2.20. Consider an integer marked pyramid with vertex $O$ and convex polygonal base $M$. If this marked pyramid is completely empty and multistory, then it is two-story. The base of the marked pyramid is integer-affine equivalent to the quadrangle $(b, 0),(0,1),(-a, 0),(0,-1)$ where $b \geqslant a \geqslant 1$. The integer-affine type of the pyramid is uniquely determined by the integers $a$ and $b($ for $b \geqslant a \geqslant 1)$ and coincides with the integer-affine type of the marked pyramid $M_{a, b}$. Two marked pyramids $M_{a, b}$ and $M_{a^{\prime}, b^{\prime}}\left(b \geqslant a \geqslant 1, b^{\prime} \geqslant a^{\prime} \geqslant 1\right)$ are integer-affine equivalent iff $a=a^{\prime}$ and $b=b^{\prime}$.

Proof. Under the assumptions of the lemma the integer distance from the two-dimensional plane containing the parallelogram $M$ to the vertex $O$ is greater than one. It follows from Proposition 2.17 that the parallelogram $M$ contains either an empty parallelogram or a parallelogram with exactly one integer point in its interior (and distinct to the vertices). By Proposition 2.18 the case of an empty parallelogram is eliminated. Consider a parallelogram $P$ with exactly one integer point inside.

Choose coordinates on the plane containing the base $M$ so that the vertices of $P$ have the following coordinates: $(1,0),(0,1),(-1,0)$, and $(0,-1)$. Note that all the coordinates of a point of this plane are integers if and only if this point is a lattice node.

Let an integer point with coordinates $(x, y)$ for some $x, y>0$ be in the base $M$. Since $M$ is convex, the point $(1,1)$ is also in $M$. This implies that the empty integer parallelogram with vertices $(0,0),(1,0),(1,1),(0,1)$ is contained in $M$. Therefore, by Proposition 2.18 the distance from the vertex of the pyramid to the twodimensional plane containing the polygon $M$ equals one.

The cases $x>0, y<0 ; x<0, y>0$; and $x, y<0$ are similar. 
Let the integer points with coordinates $(x, 0)$ and $(0, y)$, where $|x|>1$ and $|y|>1$, be in the base $M$. Then $M$ contains one of the points: $(1,1),(1,-1)$, $(-1,1)$, or $(-1,-1)$. And for the same reason, the distance from the vertex of the pyramid to the two-dimensional plane containing $M$ equals one.

Without loss of generality we suppose that $M$ does not contain points with coordinates $(0, y)$ for $|y|>1$. Then $M$ is integer-affine equivalent to the quadrangle with vertices $(b, 0),(0,1),(-a, 0),(0,-1)$, where $b \geqslant a \geqslant 1$.

Since the polygon $M$ contains the parallelogram $P$, by Lemma 2.19 the integer distance from the vertex $O$ of the marked pyramid to the two-dimensional plane containing the base $M$ equals two. The parallelogram $P$ is uniquely defined by the quadrangle with vertices $(b, 0),(0,1),(-a, 0),(0,-1)$, where $b \geqslant a \geqslant 1$ (this quadrangle contains the unique integer parallelogram with exactly one integer point distinct to the vertices). Therefore, by Lemma 2.19 the marked pyramid is integer-affine equivalent to the marked pyramid with vertex $(0,0,0)$ and base $(2,-1,0),(2,-a-1,1),(2,-1,2),(2, b-1,1)$.

The point of intersection of the quadrangular base diagonals divides the diagonals into four segments with integer lengths $1,1, a$, and $b$. Therefore the (unordered) pair of integers $[a, b]$ is an integer-affine invariant for the marked pyramid.

2.4. Proof of Theorem A for the case of triangular marked pyramids. We continue the proof by studying some special cases. Throughout this subsection we denote by $O A B C$ a triangular marked pyramid with vertex $O$ and base $A B C$.

2.4.1. Case 1: the base contains an integer polygon. Suppose that the triangle $A B C$ contains two integer points $D$ and $E$ such that the line $D E$ intersects the edges of the triangle $A B C$ and does not contain any vertex of the triangle. Without loss of generality we suppose that the open ray $D E$ with vertex at $D$ intersects $A B$, and the open ray $E D$ with vertex at $E$ intersects $B C$. Hence the triangle $A B C$ contains some integer convex quadrangle $A E D C$. By Proposition 2.17 the triangle $A B C$ contains either an integer empty parallelogram or a parallelogram integer-affine equivalent to the parallelogram with vertices $(1,0),(0,1),(-1,0)$, and $(0,-1)$.

If the triangle $A B C$ contains an integer empty parallelogram, then by Proposition 2.18 the marked pyramid $O A B C$ is single-story.

Suppose that the triangle $A B C$ does not contain an integer empty parallelogram and contains a parallelogram integer-affine equivalent to the parallelogram with vertices $(1,0),(0,1),(-1,0)$, and $(0,-1)$. Consider the coordinates on the plane containing the base such that the vertices of the above-mentioned parallelogram have the following coordinates: $(1,0),(0,1),(-1,0)$, and $(0,-1)$. If the points $(1,1),(1,-1),(-1,1)$, and $(-1,-1)$ are not contained in $A B C$, then the marked pyramid is no longer triangular. Therefore any marked pyramid of Case 1 contains some empty parallelogram, and by Proposition 2.18 it is single-story.

2.4.2. Case 2: the integer points of the base different from the vertices are not contained in one line. The only possible affine type is shown on Fig. 9.

Let us find all possible integer-affine types of such triangle. Since the triangle FED (see Fig. 9) is empty, it is integer-affine equivalent to the triangle $(1,0)$, 


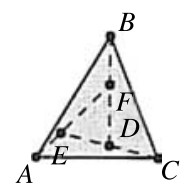

Figure 9. The affine type of triangles of Case 2

$(0,0)$, and $(0,1)$. The points $A, B$, and $C$ correspond to $(-1,0),(2,1)$, and $(0,-2)$ respectively. Hence the integer-affine type is determined in the unique way.

Lemma 2.21. Consider an integer multistory marked pyramid with vertex $O$ and triangular base $A B C$. Let $A B C$ be integer-affine equivalent to the triangle with vertices $(-2,1),(-1,-1)$, and $(1,2)$. Then the marked pyramid $O A B C$ is twostory and integer-affine equivalent to the marked pyramid $V$ of list " $M$ - $W$ ".

Proof. Let the base of an $r$-story $(r \geqslant 2)$ completely empty marked pyramid $O A B C$ be integer-affine equivalent to the triangle with vertices $(-2,1),(-1,-1)$, and $(1,2)$.

Consider the parallelepiped $P(A O B C)$ and the integer-distance coordinates corresponding to it and denoted by: $\langle x, y, z\rangle$. By Corollary 2.7 the coordinates of the vertices $O, B$, and $C$ are $\langle r, 0,0\rangle,\langle 0,7 r, 0\rangle$, and $\langle 0,0,7 r\rangle$ respectively.

Let us consider the intersection parallelogram of $P(A O B C)$ with the plane $x=1$. Now we find all lattice nodes in this parallelogram. By Proposition 2.5 there are exactly seven lattice nodes in the parallelogram of intersection. Let us describe all possible positions of these nodes in the intersection of $P(A O B C)$ with the plane $x=1$. First, there are no lattice nodes in the intersection of the marked pyramid $A O B C$ with the plane $x=1$, i.e. in the closed triangle with vertices $\langle 1,0,0\rangle,\langle 1,0,7 r-7\rangle$, and $\langle 1,7 r-7,0\rangle$. Secondly, there are no lattice nodes in all triangles obtained from the given one by applying translations by vectors $\lambda\langle 0, r, 2 r\rangle+\mu\langle 0,4 r, r\rangle$ for all integers $\lambda$ and $\mu$. On Fig. $10(r \geqslant 4)$ and Fig. 11 $(r=2,3)$ we show triangles that do not contain lattice nodes. These triangles are shaded.

So the lattice nodes of the intersection parallelogram of $P(A O B C)$ with the plane $x=1$ can be only at integer points in open triangles obtained from two triangles by translations by the vectors $\lambda\langle 0, r, 2 r\rangle+\mu\langle 0,4 r, r\rangle$ for all integers $\lambda$

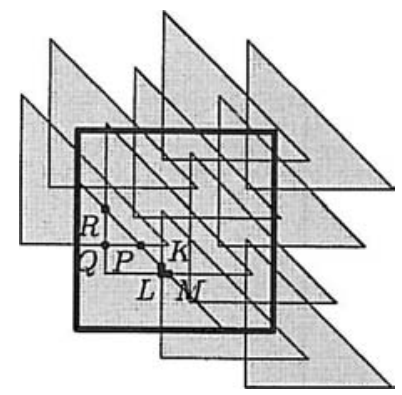

Figure 10. The intersection of $P(A O B C)$ with the plane $x=1$ (for $r>3$ ) 


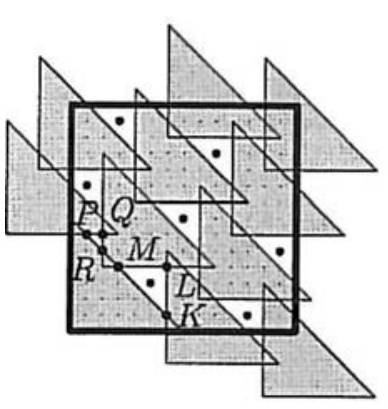

a)

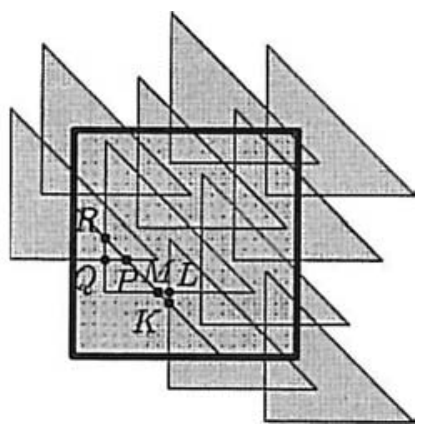

b)

Figure 11. The intersection of $P(A O B C)$ with the plane $x=1$ : a) $r=2$; b) $r=3$

and $\mu$. The vertices of the first triangle are $K\langle 1,3 r, 4 r-7\rangle, L\langle 1,3 r, 2 r\rangle$, and $M\langle 1,5 r-7,2 r\rangle$. Here the points $\langle 1,0,0\rangle$ and $L$ should be in different half-planes with respect to the line $L M$. This condition is satisfied only if $2 r>4 r-7$, i.e. $r<7 / 2$. The vertices of the second triangle are $P\langle 1,4 r-7,3 r\rangle, Q\langle 1, r, 3 r\rangle$, and $R\langle 1, r, 6 r-7\rangle$. And again the points $\langle 1,0,0\rangle$ and $Q$ should be in different halfplanes with respect to the line $P R$. This condition is satisfied only if $(4 r-7<r)$, i.e. $r<7 / 3$.

So for $r>3$ all points of the intersection parallelogram of $P(A O B C)$ with the plane $x=1$ are covered, see Fig. 10. If $r=2$, then the triangle $K L M$ contains only one integer point with coordinates $\langle 1,5,3\rangle$, see Fig. 11a). If $r=3$, then the triangle $K L M$ does not contain any integer point, see Fig. 11b).

Since the intersection parallelogram of the plane $x=1$ with the open parallelepiped should contain seven lattice nodes, the only possible case is $r=2$. There are exactly seven integer points in the complement to the union of the described triangles in the parallelogram. Hence all these points are lattice nodes. Therefore, the marked pyramid $O A B C$ is two-story and integer-affine equivalent to the marked pyramid with vertex $(0,0,0)$ and base $(2,-2,1),(2,-1,-1),(2,1,2)$ (i.e. to the pyramid $V$ of list "M-W").

It remains to study the cases of triangular pyramids with the following property. All integer points of the base of such pyramid distinct to the vertices of the pyramid are contained in some straight line passing through one of the vertices of the base triangle.

\subsubsection{Case 3: all integer points of the base distinct to vertices are contained} in a straight line - I. Suppose that all lattice nodes of the triangle $A B C$ are contained in a ray with vertex at $A$. Let the number of nodes be equal $c(c \geqslant 1)$, and also suppose that all these points are in the interior of $A B C$. Denote the nodes in the interior by $D_{1}, \ldots, D_{c}$, starting from the point closest to $A$ and increasing the indexing in the direction from $A$. It turns out that for any positive integer $c$ there exists exactly one integer-affine type of such pyramid.

Since the triangle $B D_{c} C$ is empty there exists an integer-affine transformation that takes the triangle to any other empty triangle. Let us take the triangle $B D_{c} C$ to 
the triangle $\widetilde{B} \widetilde{D}_{c} \widetilde{C}$ with vertices $(0,1),(0,0)$, and $(1,0)$ respectively. Now we determine the image of $A$. Since the point $\widetilde{D}_{c}(0,0)$ is an integer point of the triangle, the point $\widetilde{A}$ is in the third orthant $(x<0, y<0)$. Since $(-1,0)$ is not in the triangle, the point $\widetilde{A}$ is in the half-plane defined by $y<x+1$. Since $(0,-1)$ is not in the triangle, the point $\widetilde{A}$ is in the half-plane defined by $y>x-1$. Since $\widetilde{A}$ is integer, its coordinates are $(-t,-t)$ for some positive integer $t$. Since there are exactly $c$ interior integer points in the triangle $\widetilde{B} \widetilde{D}_{c} \widetilde{C}$, we have $t=c$. Therefore the triangle $\widetilde{A} \widetilde{B} \widetilde{C}$ is integer-affine equivalent to the triangle with vertices $(1,0),(0,1)$, and $(-c,-c)$.

First we study the case $c=1$.

Lemma 2.22. Consider an integer multistory marked pyramid with vertex $O$ and triangular base $A B C$. Let the triangle $A B C$ be integer-affine equivalent to the triangle with vertices $(-1,-1),(0,1)$, and $(1,0)$. Then the marked pyramid $O A B C$ is three-story and integer-affine equivalent to the marked pyramid $W$ of list " $M-W$ ".

Proof. Suppose that the base of $r$-story $(r \geqslant 2)$ completely empty marked pyramid $O A B C$ be integer-affine equivalent to the triangle with vertices $(-1,-1)$, $(0,1)$, and $(1,0)$.

Consider the parallelepiped $P(A O B C)$ and the integer-distance coordinates corresponding to it (denoted by $\langle x, y, z\rangle$ ). By Corollary 2.7 the coordinates of $O$, $B$, and $C$ equal $\langle r, 0,0\rangle,\langle 0,3 r, 0\rangle$, and $\langle 0,0,3 r\rangle$ respectively.

Let us consider the parallelogram at intersection of $P(A O B C)$ and the plane $x=1$. Now we find all lattice nodes in this parallelogram. By Proposition 2.5 there are exactly three lattice nodes in the parallelogram at intersection. Let us describe all possible positions of these nodes in the intersection of $P(A O B C)$ with the plane $x=1$. First, there are no lattice nodes in the intersection of the marked pyramid $A O B C$ with the plane $x=1$, i.e. in the closed triangle with vertices $\langle 1,0,0\rangle$, $\langle 1,0,3 r-3\rangle$, and $\langle 1,3 r-3,0\rangle$. Secondly, there are no lattice nodes in all triangles obtained from the given one by applying translations by vectors $\lambda\langle 0,3 r, 0\rangle+$ $\mu\langle 0, r, r\rangle$ for integers $\lambda$ and $\mu$. On Fig. 12, we show some triangles that do not contain lattice nodes. These triangles are shaded.

So the lattice nodes in the intersection of $P(A O B C)$ with the plane $x=1$ can be only at integer points in an open triangle obtained from the triangle $K\langle 1,3 r, r-3\rangle$,

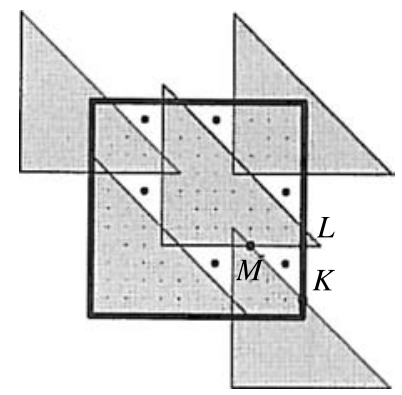

Figure 12. The intersection of $P(A O B C)$ with the plane $x=1$ 
$L\langle 1,3 r, r\rangle, M\langle 1,3 r-3, r\rangle$ by translations by vectors $\lambda\langle 0,3 r, 0\rangle+\mu\langle 0, r, r\rangle$ for any integers $\lambda$ and $\mu$. Only one point with integer coefficients $\langle 1,3 r-1, r-1\rangle$ is in the triangle $K L M$, see Fig. 12.

Shaded triangles cover almost all integer points in the intersection of $P(A O B C)$ with the plane $x=1$. Only two three-tuples of integer points are still uncovered:

1) $\langle 1,3 r-1, r-1\rangle,\langle 1, r-1,2 r-1\rangle,\langle 1,2 r-1,3 r-1\rangle$;

2) $\langle 1, r-1,3 r-1\rangle,\langle 1,2 r-1, r-1\rangle,\langle 1,3 r-1,2 r-1\rangle$.

So the lattice nodes are either the points of the first three-tuples or the points of the second one.

Suppose $\langle 1,3 r-1, r-1\rangle$ is a lattice node. (If no, then the point $\langle 1, r-1,3 r-1\rangle$ is a lattice node. Since the transformation that maps $\langle x, y, z\rangle$ to $\langle x, z, y\rangle$ is integeraffine and it preserves the parallelepiped $P(A O B C)$ and the marked pyramid $O A B C$, this case is similar.) Then the point $\langle r,(3 r-1) r,(r-1) r\rangle$ is a lattice node. Geometry of lattice nodes imply that $(3 r-1) r-(r-1) r$ is divisible by 3 . Therefore $2 r^{2}$ is divisible by 3 , and hence $r$ is also divisible by 3 .

Suppose $r=3$, then the marked pyramid exists and is integer-affine equivalent to $W$.

Let us study the case of $r=3 k$, for $k \geqslant 2$. Consider the parallelogram at intersection of $P(A O B C)$ and the plane $x=3$. Now we find all lattice nodes in this parallelogram. By Proposition 2.5 there are exactly three lattice nodes in the parallelogram of intersection. Let us describe all possible positions of these nodes. First, there are no lattice nodes in the intersection of the marked pyramid $A O B C$ with the plane $x=3$, i.e. in the closed triangle with vertices $\langle 3,0,0\rangle$, $\langle 3,3 r-9,0\rangle$, and $\langle 3,3 r-9,0\rangle$. Secondly, there are no lattice nodes in all triangles obtained from the given one by applying translations by vectors $\lambda\langle 0,3 r, 0\rangle+$ $\mu\langle 0, r, r\rangle$ for all integers $\lambda$ and $\mu$. This includes the triangle with vertices $P\langle 3,2 r, 2 r\rangle, Q\langle 3,5 r-9,2 r\rangle$, and $R\langle 3,2 r, 5 r-9\rangle$ shown on Fig. 13.

Since $\langle 1,3 r-1, r-1\rangle$ is a lattice node, the point $\langle 3,9 r-3,3 r-3\rangle$ is a lattice node. Thus $\langle 3,3 r-3,3 r-3\rangle$ is a lattice node. However, this point is in $K L M$ (for $r>1$ ) and hence $\langle 1,3 r-1, r-1\rangle$ is not a lattice node. We come to the contradiction, the case of $r=3 k$ for $k \geqslant 2$ is empty.

Lemma 2.23. Consider an integer multistory marked pyramid with vertex $O$ and triangular base $A B C$. Let the triangle $A B C$ be integer-affine equivalent to the

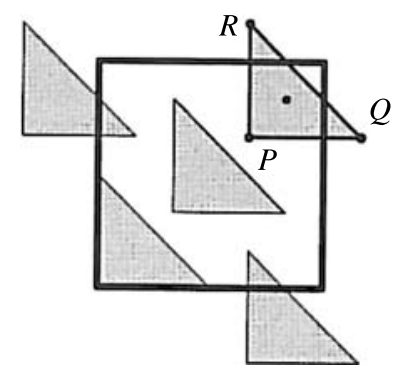

Figure 13. The intersection of $P(A O B C)$ with the plane $x=3$ 
triangle with vertices $(-c,-c),(0,-1)$, and $(-1,0)$, for $c \geqslant 2$. Then the marked pyramid $O A B C$ is not completely empty.

Proof. We prove by reductio ad absurdum. Suppose that the base of $r$-story $(r \geqslant 2)$ completely empty marked pyramid $O A B C$ is integer-affine equivalent to the triangle with vertices $(-c,-c),(0,-1)$, and $(-1,0)$, for $c \geqslant 2$. Since the triangle with vertices $(-c,-c),(1,0)$, and $(0,1)$ contains the triangle with vertices $(-1,-1),(1,0)$, and $(0,1)$, the marked pyramid $O A B C$ contains a marked subpyramid integer-affine equivalent to the pyramid of Lemma 2.22. (By a marked subpyramid $P$ of some marked pyramid $Q$ we call a convex pyramid $P$ such that the vertices of $P$ and $Q$ coincides and the base of $Q$ contains the base of $P$.) Therefore by Lemma 2.22 we have $r=3$.

Since $c \geqslant 2$, the marked pyramid $O A B C$ contains some marked subpyramid $O A^{\prime} B C$ with base $A^{\prime} B C$ integer-affine equivalent to the triangle with vertices $(-2,-2),(1,0)$, and $(0,1)$. We show now that $O A^{\prime} B C$ is not completely empty.

Consider the parallelepiped $P\left(A^{\prime} O B C\right)$ and the integer-distance coordinates corresponding to it (denoted by $\langle x, y, z\rangle$ ). By Corollary 2.7 the coordinates of $O$, $B$, and $C$ equal $\langle 3,0,0\rangle,\langle 0,15,0\rangle$, and $\langle 0,0,15\rangle$ respectively.

Let us consider the parallelogram at intersection of $P\left(A^{\prime} O B C\right)$ and the plane $x=1$. Now we find all lattice nodes in this parallelogram. First, there are no lattice nodes in the intersection of the marked pyramid $A^{\prime} O B C$ with the plane $x=1$, i.e. in the closed triangle with vertices $\langle 1,0,0\rangle,\langle 1,0,12\rangle$, and $\langle 1,12,0\rangle$. Secondly, there are no lattice nodes in all triangles obtained from the given one by applying translations by vectors $\lambda\langle 0,15,0\rangle+\mu\langle 0,3,3\rangle$ for all integers $\lambda$ and $\mu$. These triangles contain all integer points of the intersection of $P\left(A^{\prime} O B C\right)$ with the plane $x=1$, see Fig. 14 .

So, the marked pyramid $O A^{\prime} B C$ is not completely empty. Hence the marked pyramid $O A B C$ is not completely empty. Thus $r \neq 3$. We come to the contradiction.

2.4.4. Case 4: all integer points of the base distinct to vertices are contained in a straight line - II. Suppose that all integer points of the triangle $A B C$ are contained in the ray with vertex $A$. Let the number of points equal $b(b \geqslant 1)$, and the

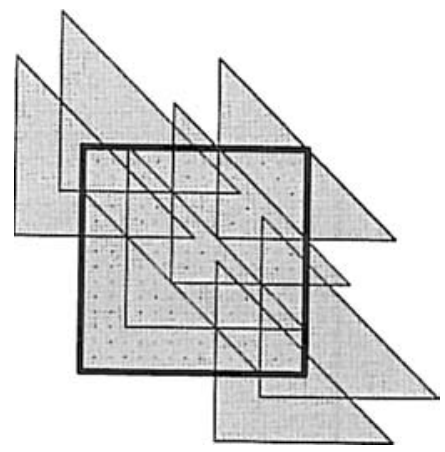

Figure 14. The intersection of $P\left(A^{\prime} O B C\right)$ with the plane $x=1$ 
last point be in the edge $B C$. Denote these points by $D_{1}, \ldots, D_{b}$, starting from the point closest to $A$ and increasing the indexing in the direction from $A$. It turns out that for any $b$ there exists exactly one integer-affine type of such pyramid.

Since the triangle $D_{b} D_{b-1} B$ is empty there exists an integer-affine transformation that takes the triangle to any other empty triangle. We take the triangle $D_{b} D_{b-1} B$ to the triangle with vertices $(0,0),(1,0)$, and $(0,-1)$ respectively. Then $C$ maps to $(0,1)$, and $A$ maps to $(b, 0)$. Therefore the triangle $A B C$ is integer-affine equivalent to the triangle with vertices $(0,-1),(b, 0)$, and $(0,1)$.

First we study the case $b=2$.

Lemma 2.24. Consider an integer multistory marked pyramid with vertex $O$ and triangular base $A B C$. Let the triangle $A B C$ be integer-affine equivalent to the triangle with vertices $(2,0),(0,-1)$, and $(0,1)$. Then the marked pyramid $O A B C$ is two-story and integer-affine equivalent to the marked pyramid $U_{2}$ of list " $M$ - $W$ ".

Proof. Suppose that the base of $r$-story $(r \geqslant 2)$ completely empty marked pyramid $O A B C$ be integer-affine equivalent to the triangle with vertices $(2,0)$, $(0,-1)$, and $(0,1)$.

Consider the parallelepiped $P(A O B C)$ and the integer-distance coordinates corresponding to it (denoted by $\langle x, y, z\rangle$ ). By Corollary 2.7 the coordinates of $O$, $B$, and $C$ equal $\langle r, 0,0\rangle,\langle 0,4 r, 0\rangle$, and $\langle 0,0,4 r\rangle$ respectively.

Consider the parallelogram at intersection of $P(A O B C)$ and the plane $x=1$. Now we find all lattice nodes in this parallelogram. By Proposition 2.5 there are exactly three lattice nodes in the parallelogram at intersection. Let us describe all possible positions of these nodes. First, there are no lattice nodes in the intersection of the marked pyramid $A O B C$ with the plane $x=1$, i.e. in the closed triangle with vertices $\langle 1,0,0\rangle,\langle 1,0,4 r-4\rangle$, and $\langle 1,4 r-4,0\rangle$. Secondly, there are no lattice nodes in triangles obtained from the given one by applying translations by vectors $\lambda\langle 0,4 r, 0\rangle+\mu\langle 0, r, r\rangle$ for all integers $\lambda$ and $\mu$. We show (shaded) triangles that do not contain lattice nodes on Fig. 15.

So the lattice nodes in the intersection of $P(A O B C)$ with the plane $x=1$ can be only at integer points in an open triangle obtained from the triangle $K\langle 1,4 r, 2 r-3\rangle$, $L\langle 1,4 r, 2 r\rangle, M\langle 1,4 r-3,2 r\rangle$ by translations by vectors $\lambda\langle 0,4 r, 0\rangle+\mu\langle 0, r, r\rangle$ for all integers $\lambda$ and $\mu$ and the symmetry about the plane $y=z$. Only the points with

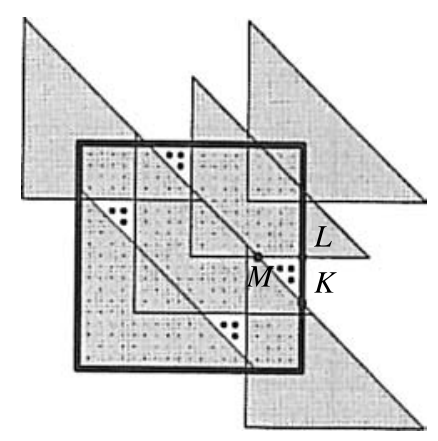

Figure 15. The intersection of $P(A O B C)$ with the plane $x=1$ 
integer coordinates $\langle 1,4 r-2,2 r-1\rangle,\langle 1,4 r-1,2 r-1\rangle$, and $\langle 1,4 r-1,2 r-2\rangle$ are in the triangle $K L M$, see Fig. 15.

We prove that one of these points is a lattice node by reductio ad absurdum. Suppose that the triangle $K L M$ does not contain a lattice node. Then there are no lattice nodes in triangles obtained from $K L M$ by applying translations by vectors of the form $\lambda\langle 0,4 r, 0\rangle+\mu\langle 0, r, r\rangle$ for all integers $\lambda$ and $\mu$. Hence the intersection of the parallelepiped $P(A O B C)$ with the plane $x=1$ does not contain integer nodes. We come to the contradiction. So one of the points $\langle 1,4 r-2,2 r-1\rangle$, $\langle 1,4 r-1,2 r-1\rangle$, and $\langle 1,4 r-1,2 r-2\rangle$ is a lattice node.

Suppose that $r \geqslant 3$ and consider the plane $x=2$. First, there are no lattice nodes in the intersection of the marked pyramid $A O B C$ with the plane $x=2$, i.e. in the closed triangle with vertices $\langle 1,0,0\rangle,\langle 1,0,4 r-8\rangle$, and $\langle 1,4 r-8,0\rangle$. Secondly, there are no lattice nodes in all triangles obtained from the given one by applying translations by vectors $\lambda\langle 0,4 r, 0\rangle+\mu\langle 0, r, r\rangle$ for all integers $\lambda$ and $\mu$. In particular, there are no lattice nodes in the triangle with vertices $P\langle 2,3 r, 3 r\rangle$, $Q\langle 2,7 r-8,3 r\rangle$, and $R\langle 2,3 r, 7 r-8\rangle$.

Suppose that the point $\langle 1,4 r-2,2 r-1\rangle,\langle 1,4 r-1,2 r-1\rangle$, or $\langle 1,4 r-1$, $2 r-2\rangle$ is a lattice node, then $\langle 2,8 r-4,4 r-2\rangle,\langle 2,8 r-2,4 r-2\rangle$, or $\langle 2,8 r-2$, $4 r-4\rangle$ respectively is also a lattice node. Hence the point $\langle 2,4 r-4,4 r-2\rangle$, $\langle 2,4 r-2,4 r-2\rangle$, or $\langle 2,4 r-2,4 r-4\rangle$ respectively is a lattice node. The last three points are contained in the triangle $P Q R$ with vertices $P\langle 2,3 r, 3 r\rangle$, $Q\langle 2,7 r-8,3 r\rangle$, and $R\langle 2,3 r, 7 r-8\rangle$, for $r>3$ (see Fig. 16), and hence these points are not lattice nodes. For $r=3$, the point $\langle 1,11,5\rangle$ is not a lattice node by the same reason. The points $\langle 1,10,5\rangle$ and $\langle 1,11,4\rangle$ are not lattice nodes, since the points $\langle 3,30,15\rangle$ and $\langle 3,33,12\rangle$ are not lattice nodes of the plane $x=3$ (all such node coordinates are $\langle 3,4 m, 4 n\rangle$ for some integers $m$ and $n$ ). From the above we conclude that $r \leqslant 2$.

Suppose now that $r=2$ and consider the points $\langle 1,6,4\rangle,\langle 1,7,3\rangle$, and $\langle 1,7,4\rangle$. The points $\langle 1,6,4\rangle$ and $\langle 1,7,3\rangle$ are not lattice nodes, since the points $\langle 2,12,6\rangle$ and $\langle 2,14,8\rangle$ are not lattice nodes of the plane $x=2$ (all such nodes coordinates are $\langle 2,4 m, 4 n\rangle$ for some integers $m$ and $n$ ). The point $\langle 1,7,4\rangle$ defines a uniquepossible integer-affine type of marked pyramids with such base - the integer-affine type of the marked pyramid $U_{2}$.

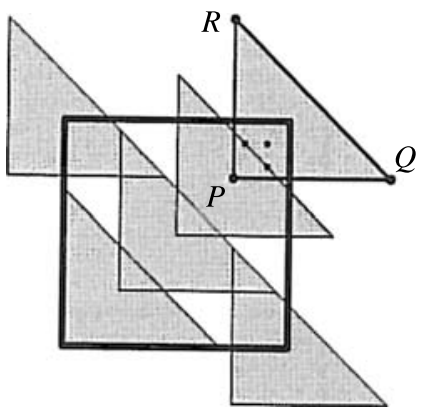

Figure 16. The intersection of $P(A O B C)$ with the plane $x=2$ 
Now we will study the general case $(b \geqslant 2)$.

Lemma 2.25. Consider an integer multistory marked pyramid with vertex $O$ and triangle base $A B C$. Let the triangle $A B C$ be integer-affine equivalent to the triangle with vertices $(b, 0),(0,-1)$, and $(0,1)$, for $b \geqslant 2$. Then the marked pyramid $O A B C$ is two-story and integer-affine equivalent to the marked pyramid $U_{b}$ of list " $M-W$ ".

Proof. Let the base of $r$-story $(r \geqslant 2)$ completely empty marked pyramid $O A B C$ be integer-affine equivalent to the triangle with vertices $(b, 0),(0,-1)$, and $(0,1)$.

Since the triangle with vertices $(b, 0),(0,-1)$, and $(0,1)$ contains the triangle with vertices $(2,0),(0,-1)$, and $(0,1)$, the marked pyramid $O A B C$ contains a marked subpyramid that is integer-affine equivalent to a marked pyramid of Lemma 2.24. Since the subpyramid is completely empty, by Lemma 2.24 we have that it is two-story.

Suppose now $r=2$. Consider the parallelepiped $P(A O B C)$ and the integerdistance coordinates corresponding to it (denoted by $\langle x, y, z\rangle$ ). By Corollary 2.7 the coordinates of $O, B$, and $C$ equal $\langle 2,0,0\rangle,\langle 0,4 b, 0\rangle$, and $\langle 0,0,4 b\rangle$ respectively.

Consider the parallelogram at the intersection of $P(A O B C)$ and the plane $x=1$. Now we find all lattice nodes in this parallelogram. By Proposition 2.5 there are exactly $2 b$ lattice nodes in the parallelogram at intersection. Let us describe all possible positions of these nodes. First, there are no lattice nodes in the intersection of the marked pyramid $A O B C$ with the plane $x=1$, i.e. in the closed triangle with vertices $\langle 1,0,0\rangle,\langle 1,0,2 b\rangle$, and $\langle 1,2 b, 0\rangle$. Secondly, there are no lattice nodes in all triangles obtained from the given one by applying translations by vectors $\lambda\langle 0,4 b, 0\rangle+\mu\langle 0,2,2\rangle$ for all integers $\lambda$ and $\mu$. We show some (shaded) triangles that do not contain any lattice nodes on Fig. 17.

So the lattice nodes of the intersection of $P(A O B C)$ with the plane $x=1$ can be only at integer points in an open triangle obtained from the triangle $K\langle 1,4 b, 2 b-4\rangle, L\langle 1,4 b, 2 b\rangle, M\langle 1,4 b-4,2 b\rangle$ by translations by vectors $\lambda\langle 0,4 b, 0\rangle+\mu\langle 0,2,2\rangle$ for all integers $\lambda$ and $\mu$ and the symmetry about the plane $y=z$. Only the points with integer coefficients $\langle 1,4 b-2,2 b-1\rangle,\langle 1,4 b-1$,

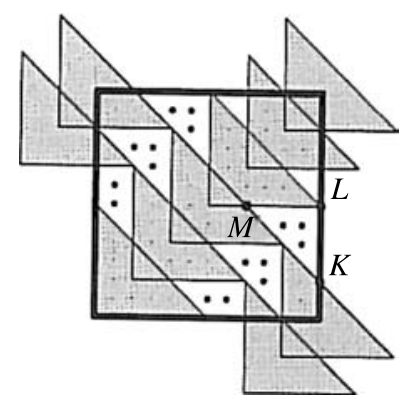

Figure 17. The intersection of $P(A O B C)$ with the plane $x=1$ 
$2 b-1\rangle$, and $\langle 1,4 b-1,2 b-2\rangle$ are in the triangle $K L M$ (the case $b=3$ is shown in Fig. 17).

One of the integer points of this triangle is a lattice node (the other uncovered parts of the section can be obtained by translations by vectors $\lambda\langle 0,4 b, 0\rangle+$ $\mu\langle 0,2,2\rangle$ for integers $\lambda$ and $\mu$ ).

Consider the plane $x=2$. The point $\langle 2, y, z\rangle$ is a lattice node iff there exist integers $m$ and $n$ such that $z=2 m$, and $y=2 m+2 b n$.

We show that the point $\langle 1,4 b-2,2 b-1\rangle$ is not a lattice node by reductio ad absurdum. Suppose that this point is a lattice node. Then the point $\langle 2,8 b-4$, $4 b-2\rangle$ is also a lattice node. Let us find integers $m$ and $n$ such that $4 b-2=2 m$ and $8 b-4=2 m+2 b n$. Then $m=2 b-1, n=\frac{2 b-1}{b}$. If $b \geqslant 2$, then $n$ is not integer. We come to the contradiction. Therefore the point $\langle 1,4 b-2,2 b-1\rangle$ is not a lattice node.

By the same reasons the point $\langle 1,4 b-1,2 b-2\rangle$ is not a lattice node. The last point of the triangle $\langle 1,4 b-1,2 b-1\rangle$ determines the pyramid of the integeraffine type $U_{b}$.

2.4.5. Case 5: integer points of the base distinct to the vertices are contained in one edge of the base. It remains to study the case of the last most simple series of triangular marked pyramids. Suppose that all integer points of the base $A B C$ distinct to the vertices are contained in $A C$, and the integer length of $A C$ is $a-1$, for some $a \geqslant 2$. The case of $a=1$ is the case of empty marked pyramid, it was studied before in Corollary 2.13. Denote these points by $D_{1}, \ldots, D_{a-1}$ starting from the point closest to $A$ and increasing the indexing in the direction to $C$.

Consider an integer multistory marked pyramid with vertex $O$ and triangular base $A B C$. Let the triangle $A B C$ be integer-affine equivalent to the triangle with vertices $(0,0),(0,1)$, and $(a, 0)$, for $a \geqslant 2$.

Lemma 2.26. The marked pyramid $O A B C$ is integer-affine equivalent to the marked pyramid of the following list.

List " $T$ ":

$-T_{a, 1}^{0}$;

- $T_{a, r}^{\xi}$, where $\xi$ and $r$ are relatively prime and satisfy: $r \geqslant 2$ and $0<\xi \leqslant r / 2$.

All integer marked pyramids listed in "T" are completely empty and integerlinear nonequivalent to each other.

Proof. 1. Preliminary statement. Let us show that the marked pyramid $O A B C$ is integer-affine equivalent to the marked pyramid $T_{a, r}^{\xi}$, for some positive integer $\xi \leqslant r / 2$.

First of all two single-story marked pyramids with the same $a$ are integer-affine equivalent, since the integer points of the edges of the pyramid generate all integer lattice.

Let the base of $r$-story $(r \geqslant 2)$ completely empty marked pyramid $O A B C$ be integer-affine equivalent to the triangle with vertices $(0,0),(0,1)$, and $(a, 0)$. Consider the parallelepiped $P\left(A O B D_{1}\right)$ and the integer-distance coordinates cor- 
responding to it (denoted by $\langle x, y, z\rangle$ ). By Corollary 2.7 the coordinates of $O, B$, and $C$ equal $\langle r, 0,0\rangle,\langle 0, r, 0\rangle$, and $\langle 0,0, r\rangle$ respectively.

By Corollary 2.9 (since the tetrahedron $A O B D_{1}$ is empty) all interior lattice nodes are contained in one of three diagonal planes: $x+z=r, y+z=r$, or $x+y=r$. Now we examine all the cases.

Let all interior lattice nodes be contained in the plane $x+z=r$. By Lemma 2.6 there exists exactly one lattice node $K$ contained in the plane $x=1$. So, $K$ is in the intersection of these two planes, and its coordinates are $\langle 1, \xi, r-1\rangle$, where $0<\xi<r$. Now we come back to the old coordinates associated with the lattice. Since the integer distance from $K$ to the two-dimensional plane containing the face $A D_{1} B$ equals one, the tetrahedron $A D_{1} B K$ can be taken by some integer-affine transformation to the tetrahedron with vertices $(0,0,0)$, $(1,0,0),(0,1,0)$, and $(0,0,1)$. By such transformation the vertex $O$ maps to $(-\xi, 1-r, r)$, and $C$ maps to $(a, 0,0)$. Let us translate the obtained pyramid by the integer vector $(\xi, r-1, r)$. Finally we get the marked pyramid $T_{a, r}^{\xi}$. Hence the marked pyramid $O A C B$ is integer-affine equivalent to the marked pyramid $T_{a, r}^{\xi}$, where $0<\xi<r$. Consider the integer-affine transformation taking the points $O, A, B, C$ to the points $O, C, B, A$ respectively, then the point $K$ maps to the point $(r-\xi, 1-r, r)$. Choose the smallest one of $\xi$ and $r-\xi$. Obviously, this number is not greater than $r / 2$.

Let all interior lattice nodes be contained in the plane $y+z=r$ in the integerdistance coordinate system. By Lemma 2.6 there exists exactly one lattice node $K$ contained in the plane $x=1$. So, $K$ is in the intersection of these two planes, and its coordinates are $\langle 1, \xi, r-\xi\rangle$, where $0<\xi<r$. The intersection of the marked pyramid $O A B C$ with the plane $x=1$ is a triangle with vertices $\langle 1,0,0\rangle$, $\langle 1, a r-a, 0\rangle$, and $\langle 1,0, r-1\rangle$. This triangle contains all integer points $\langle 1, t, r-t\rangle$, for $2 \leqslant t \leqslant r$. Hence $\xi=1$. Therefore the point $K$ is in the plane $x+z=r$, so, we are in the position of the previous case.

Let all interior lattice nodes be contained in the plane $x+y=r$ in the integerdistance coordinate system. By Lemma 2.6 there exists exactly one lattice node $K$ contained in the plane $z=1$. So, $K$ is in the intersection of these two planes, and its coordinates are $\langle\xi, r-\xi, 1\rangle$, where $0<\xi<r$. The intersection of the marked pyramid $O A B C$ with the plane $z=1$ is a triangle with vertices $\langle 0,0,1\rangle$, $\langle r-1,0,1\rangle$, and $\langle 0, a r-a, 1\rangle$. This triangle contains all integer points $\langle t, r-t, 1\rangle$, for $1 \leqslant t \leqslant r-1$. Hence $\xi=r-1$. Therefore the point $K$ is again in the plane $x+z=r$.

So, the marked pyramid $O A B C$ is integer-affine equivalent to a marked pyramid $T_{a, r}^{\xi}$ for some positive integer $\xi \leqslant r / 2$.

2. Completeness of list " $T$ " and completely emptiness of the marked pyramids of " $T$ ". Let us show that the marked pyramids $T_{a, r}^{\xi}$ of the list " $T$ " are completely empty. Denote the vertices of the marked pyramids by $O, A, B, C$, and the integer points of $A C$ by $D_{i}$.

Denote also the point $A$ by $D_{0}$, and the point $C$ by $D_{a}$. Note that the marked pyramid $O D_{i} D_{i+1} B$ is integer-affine equivalent to the marked pyramid $P_{r}^{\xi}$, for any positive integer $i \leqslant a$, since the marked pyramid $O D_{i} D_{i+1} B$ can be obtained from the pyramid $P_{r}^{\xi}$ by applying the compositions of the integer-linear transformation 
defined by the following matrix

$$
\left(\begin{array}{ccc}
\xi+i+1 & \xi+i & -\xi-i \\
r-1 & r-1 & 2-r \\
-r & -r & r-1
\end{array}\right)
$$

and the translation by the integer vector $(-\xi, 1-r, r)$.

By Corollary 2.11 if $\xi$ and $r$ are relatively prime, then the marked pyramids $O A D_{1} B, O D_{1} D_{2} B, \ldots, O D_{a-1} C B$ are empty, and hence their union $O A B C$ is completely empty.

By the same reasons the marked pyramids $T_{a, r}^{\xi}$ with relatively prime $\xi$ and $r$ are completely empty.

Therefore list " $T$ " is complete, and all listed pyramids are completely empty.

3. Irredundance of list " $T$ ". Now we prove that all marked pyramids $T_{a, r}^{\xi}$ of list " $T$ " are integer-affine nonequivalent to each other. Obviously, that the marked pyramids with different $a$ are nonequivalent. Since the integer distance from the marked vertex to the two-dimensional plane of the marked base is an integer-affine invariant, the marked pyramids with distinct $r$ are nonequivalent.

For the case of pyramids with the same integers $a>1$ and $r$ we construct the following integer-linear invariant. Consider an arbitrary marked pyramid $O A B C$, where all its lattice nodes are contained in the edge $A C$. As it was shown before the empty marked pyramids $O A D_{1} B, O D_{1} D_{2} B, \ldots, O D_{a-1} C B$ are integer-affine equivalent to the marked pyramid $P_{r}^{\xi}$ with $0 \leqslant \xi \leqslant r / 2$. Since the collection of this marked pyramids is defined in a unique way and by Corollary 2.11, the type of such $P_{r}^{\xi}$ is an invariant. This invariant distinguishes different marked pyramids of list " $T$ ".

So, we have studied all possible cases of integer-affine types of multistory completely empty convex three-dimensional marked pyramids. It remains to say a few words about the irredundance of list " $\mathrm{M}-\mathrm{W}$ " of Theorem A.

2.4.6. Irredundance of list " $M-W$ ". If two marked pyramids have integeraffine nonequivalent bases, then these pyramids are also integer-affine nonequivalent. The integer-affine types of the base distinguish almost all marked pyramids of list "M-W". This does not work only for pyramids $T_{a, r}^{\xi}$ with the same $a$ and $r$, and distinct $\xi$ from list "M-W". Such pyramids $T_{a, r}^{\xi}$ are integer-affine nonequivalent by Lemma 2.26 (see list " $T$ ").

The proof of the main theorem is completed.

\section{Proof of Theorem B}

3.1. Completeness of lists " $\alpha_{n}$ " for $n \geqslant 2$ of Theorem B. Consider some marked pyramid with marked vertex at the origin and some compact two-dimensional face of a sail as base. It follows from the definition of multidimensional continued fractions that such pyramid is completely empty.

Lemma 3.1. Two two-dimensional faces are integer-linear equivalent iff the corresponding completely empty marked pyramids are integer-affine equivalent. 


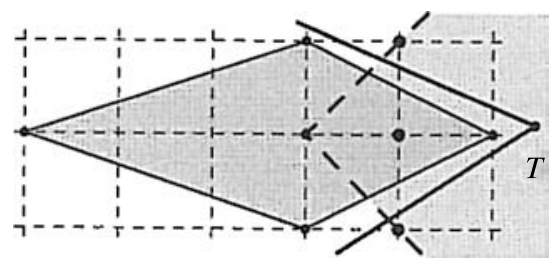

Figure 18. One of the vertices of $T$ is in the shaded (open) angle

The proof of this lemma is straightforward and we leave it for the reader.

Lemma 3.1 and Theorem A (see list "M-W") imply that for any $n>2$, list " $\alpha_{n}$ " of Theorem B is complete. Now we study the case of two-dimensional continued fractions. By Theorem A the list of all triangular faces in list " $\alpha_{2}$ " is complete.

Lemma 3.2. Any two-dimensional continued fraction does not contain faces that are integer-linear equivalent to the quadrangle with vertices $(2,-1,0)$, $(2,-a-1,1),(2,-1,2),(2, b-1,1)$ for $b \geqslant a \geqslant 1$.

Proof. We prove by reductio ad absurdum. Suppose that there exists a twodimensional continued fraction with a face $F$ integer-linear equivalent to the quadrangle with vertices $(2,-1,0),(2,-a-1,1),(2,-1,2),(2, b-1,1)$ for $b \geqslant a \geqslant 1$. Consider coordinates on the plane containing $F$ such the coordinates of the vertices of $F$ are $(a, 0),(0,1),(-b, 0)$, and $(0,-1)$. Note that the point in this plane is a lattice node iff its new coordinates are integers.

The points $(1,1),(1,-1),(-1,1)$, and $(-1,-1)$ are in the complement to $F$. Three planes of the two-dimensional continued fraction intersect with the plane containing $F$ at three lines. The face $F$ is in the interior of the triangle $T$ generated by the intersection lines. The triangle $T$ contains $F$, and the set $T \backslash F$ does not contain any integer point. Notice that the point $(1,0)$ is in $F$, and the points $(1,1)$ and $(1,-1)$ are not in $F$. Note also that the points $(1,0),(1,1)$, and $(1,-1)$ are in one straight line. Then the open angle with vertex $(0,0)$ and edges passing through the points $(1,1)$, and $(1,-1)$, contains some vertex of the triangle $T$, see Fig. 18 .

The same holds for two adjacent angles and for the opposite angle. Therefore the triangle $T$ has at least four vertices. We come to the contradiction.

The above lemmas yield the completeness of list " $\alpha_{n}$ " for any dimension $n \geqslant 2$.

\subsection{Realizability and nonequivalence of faces.}

Lemma 3.3. For any $n \geqslant 2$, any face of list " $\alpha_{n}$ " is realizable. Any two different faces of this list are integer-linear nonequivalent to each other.

Proof. i) First, let us show that any triangular face (denote it by $A B C$ ) of list " $\alpha_{2}$ " is realizable. Consider the continued fractions $\Omega^{2}$ defined by three planes containing the segments $A B, B C$, and $A B$ respectively. It is obvious, that $\Omega^{2}$ contains $A B C$ as a face.

ii) Second, we show how to realize a quadrangular face (denote it by $A B C D$ ) of list " $\alpha_{3}$ ". We remind that $A B C D$ lie in the plane $a_{4}=0$ in the coordinates 
$\left(a_{1}, a_{2}, a_{3}, a_{4}\right)$. Let $O$ be the origin, $P$ denote the intersection of the diagonals of $A B C D$, and $E=(0,0,0,1)$. Denote also by $|W R|$ the Euclidean distance between the points $W$ and $R$. Denote

$$
\begin{aligned}
& K=B+\overline{P A}+\varepsilon|P A||P B| \overline{O E}, \quad L=B+\overline{P C}-\varepsilon|P C||P B| \overline{O E}, \\
& N=B+\overline{P A}-\varepsilon|P A||P D| \overline{O E}, \quad M=B+\overline{P C}+\varepsilon|P C||P D| \overline{O E},
\end{aligned}
$$

for a small positive $\varepsilon$. The symplex $K L M N$ intersects the plane $a_{4}=0$ by $A B C D$. If we chose $\varepsilon$ small enough then the symplex $O K L M N$ contains only the lattice nodes of the plane $a_{4}=0$, i.e. the nodes of $A B C D$. Therefore the three-dimensional continued fraction defined by four planes containing faces $O K L M, O K L N$, $O K M N$, and $O L M N$ contains $A B C D$ as a face.

iii) Suppose now some $(n-1)$-dimensional continued fraction $\Omega^{n-1}$ contains a face $F$. Let us construct an $n$-dimensional continued fraction $\Omega^{n}$ containing $F$. Suppose $\Omega^{n-1}$ is defined by the planes $l_{i}\left(a_{1}, \ldots, a_{n}\right)=0$, for $i=1, \ldots, n$. Consider than the $n$-dimensional continued fraction $\Omega^{n}$ defined by the planes $l_{i}\left(a_{1}, \ldots, a_{n}\right)=0$ for $i=1, \ldots, n$ and an additional plane $a_{n+1}=0$. It is clear that $\Omega^{n}$ contains all the faces of $\Omega^{n-1}$. In particular, $F$ is a face of $\Omega^{n}$.

iv) From i) it follows that the faces of list " $\alpha_{2}$ " are realizable. This together with ii) and iii) imply that the faces of list " $\alpha_{3}$ " are realizable. Finally, iv) inductively implies that all Lists " $\alpha_{n}$ " for $n \geqslant 5$ are realizable.

v) Nonequivalence follows directly from Lemma 3.1 and Theorem A.

Remark 3.4. Actually a more general statement holds. The set of all continued fractions containing any face of list " $\alpha_{n}$ " is open in the natural topology on the set of all $n$-dimensional continued fractions.

Lemmas 3.2 and 3.3 conclude the proof of Theorem B.

Acknowledgements. The author is grateful to professor V. I. Arnold, professor A. B. Sossinsky, and E. I. Korkina for constant attention to this work and useful remarks and discussions, and Université Paris-Dauphine - CEREMADE and Mathematisch Instituut Universiteit Leiden for the hospitality and excellent working conditions.

\section{References}

[1] Arnold VI (1989) A-graded algebras and continued fractions. Comm Pure Appl Math 142: 993-1000

[2] Arnold VI (2002) Continued Fractions. Moscow: Moscow Center of Continuous Math Education

[3] Arnold VI (1999) Preface. Amer Math Soc Trans 197: ix-xii

[4] Arnold VI (1998) Higher dimensional continued fractions. Regular Chaotic Dynamics 3: $10-17$

[5] Briggs K (2002) Klein polyhedra. http://keithbriggs.info/klein-polyhedra.html

[6] Bryuno AD, Parusnikov VI (1994) Klein polyhedrals for two cubic Davenport forms. Math Notes 56: $9-27$

[7] Bryuno AD, Parusnikov VI (1997) Comparison of different generalizations of continued fractions. Math Notes 61: 339-348

[8] German ON (2002) Sails and Hilbert bases. Proc Steklov Inst Math 239: 88-95

[9] Hermite C (1839) Letter to C. D. J. Jacobi. J Reine Angew Math 40: 286

[10] Hinchin AYa (1961) Continued fractions. FISMATGIS

[11] Hirzebruch F (1953) Über vierdimensionale Riemannsche Flächen mehrdeutiger analytischer Funktionen von zwei komplexen Veränderlichen. Math Ann 126: 1-22 
[12] Jung HWE (1908) Darstellung der Funktionen eines algebraischen Körpers zweier unabhängigen Veränderlichen $x, y$ in der Umgebung einer Stelle $x=a, y=b$. J Reine Angew Math 133: 289-314

[13] Karpenkov O (2004) On tori decompositions associated with two-dimensional continued fractions of cubic irrationalities. Funct Anal Appl 38: 28-37

[14] Karpenkov ON (2004) On two-dimensional continued fractions for integer hyperbolic matrices with small norm. Russian Math Surveys 59: 149-150

[15] Karpenkov O (2005) Classification of three-dimensional multistory completely empty convex marked pyramids. Russian Math Surveys 60: $169-170$

[16] Karpenkov $\mathrm{O}$ (2006) On existence and uniqueness conditions of the lattice triangle with the given angles. Russian Math Surveys 61: 185-186

[17] Klein F (1895) Über eine geometrische Auffassung der gewöhnliche Kettenbruchentwicklung. Nachr Ges Wiss Göttingen Math-Phys K1 3: 357-359

[18] Klein F (1896) Sur une représentation géométrique de développement en fraction continue ordinaire. Nouv Ann Math 15: 327-331

[19] Kontsevich ML, Suhov YuM (1999) Statistics of Klein polyhedra and multidimensional continued fractions. Amer Math Soc Trans 197: 9-27

[20] Korkina EI (1993) The simplest 2-dimensional continued fraction. J Math Sci, New York 82: $3680-3685$

[21] Korkina EI (1994) La périodicité des fractions continues multidimensionelles. C R Acad Sci Paris 319: $777-780$

[22] Korkina EI (1995) Two-dimensional continued fractions. The simplest examples. Proc Steklov Math Inst 209: 143-166

[23] Korkina EI (1996) The simplest 2-dimensional continued fraction. J Math Sci 82: 3680-3685

[24] Lachaud G (1993) Polyèdre d'Arnold et voile d'un cône simplicial: analogues du thèoreme de Lagrange. C R Acad Sci Paris 317: 711-716

[25] Lachaud G (1995) Voiles et Polyèdres de Klein. Preprint n 95-22, Laboratoire de Mathématiques Discrètes du C.N.R.S., Luminy

[26] Mittal AK, Gupta AK (2000) Bifurcating Continued Fractions. http://www.arxiv.org/ftp/math/ papers/0002/0002227.pdf

[27] Moussafir J-O (2000) Sales and Hilbert bases. Func Anal Appl 34: 43-49

[28] Moussafir J-O (2000) Voiles et Polyédres de Klein: Geometrie, Algorithmes et Statistiques. Thése, Université Paris IX - Dauphine, http://www.ceremade.dauphine.fr $/{ }^{-} \mathrm{msfr} / \operatorname{articles}$ _msfr/these. ps.gz

[29] Minkowski H (1896) Généralisation de le théorie des fractions continues. Ann Sci Ecole Norm Super ser III 13: 41-60

[30] Okazaki R (1993) On an effective determination of a Shintani's decomposition of the cone $\mathbb{R}_{+}^{n}$. J Math Kyoto Univ 33-34: 1057-1070

[31] Parusnikov VI (1995) Klein's polyhedra for the third extremal ternary cubic form. Preprint 137 of Keldysh Institute of the RAS, Moscow

[32] Parusnikov VI (2000) Klein's polyhedra for the fourth extremal cubic form. Mat Zametki 67: $110-128$

[33] Shmoilov VI (2003) Continued fractions: the bibliography. L'vov: Merkator

[34] Skorobogat'ko VYa (1982) Branching continued fractions and their application in computational mathematics. Theoretical and applied problems of computational mathematics unpublished manuscript

[35] Tsuchihashi H (1973) Higher dimensional analogues of periodic continued fractions and cusp singularities. Tohoku Math J 35: 176-393

[36] Voronoi GF (1952) On one generalization of continued fraction algorithm. USSR Acad Sci 1: 197-391

[37] White GK (1964) Lattice tetrahedra. Canadian J Math 16: 389-396

Author's address: Mathematical Institute, Leiden University, P.O. Box 9512, 2300 RA Leiden, The Netherlands, e-mail: karpenk@mccme.ru 